Management Forschung und Praxis

Universität Konstanz

herausgegeben von Prof. Dr. Rüdiger G. Klimecki

Rüdiger G. Klimecki und Gilbert Probst*

\title{
Interkulturelles Lernen
}

Nr. 4 (1992)

Diskussionsbeitrag Juli 1992

Rüdiger Klimecki, Prof. Dr.

Gilbert Probst, Prof. Dr.

Lehrstuhl für Management

Lehrstuhl für Unternehmungs-

Fakultät für Verwaltungswissenschaft

organisation und Management

Universiät Genf

2, rue de Candolle

Postfach $5560<$ D 493>

$\mathrm{CH}-1211$ Genf

Tel. (07531) 882394

Tel. (022) 7057254

e-mail: Ruediger.G.Klimecki@popserver.uni-konstanz.de

Dieser Beitrag erscheint in: Haller, M./Pleitner, H.J./Bleicher, K./Wunderer, R. (Hrsg.), Globalisierung der Wirtschaftseinwirkungen auf die Betriebswirtschafts-lehre. 54. Wissenschaftliche Jahrestagung des Verbandes der Hochschullehrer für Betriebswirtschaft. St. Gallen. Haupt Verlag. Sommer .1993

* Die Autoren danken M. Oliver Altehage und Bettina Büchel für ihre proaktive Mitarbeit und Diskursbereitschaft bei der Erstellung dieses Beitrages. 


\section{Überblick:}

Der nachfolgende Beitrag beschäftigt sich mit der Frage, wie die Wettbe

werbs fähigkeit auf globalen Märkten durch institutionales Lernen ver bessert werden kann. Dazu wird ein Konzept des "interkulturellen organi sationalen Lernens" vorgeschlagen. Aus der Verbindung des Kulturan satzes mit Konzepten zur "lern fähigen Organisation" ergeben sich neue Sichtweisen für die Entwicklung der organisationalen Handlungskom petenz im Kontext der Globalisierung.

Die Bedeutung eines lernorientierten Managements mit interkultureller Aus richtung ergibt sich aus vielfältigen Kulturdivergenzen und den daraus resul die es im Rahmen von Globalisie rungsprozessen zu bew ältigen gilt. Um die zur globalen Wettbewerbsfähigkeit notwendigen Problemlösungs verbessern und, wenn möglich, zu ei nem eigenständigen Erfolgs kompe tenzen zu müssen Lernprozesse eingeleitet werden, mit denen die Informationsbasis als auch die Wertstrukturen der Organi sation mit den Anfor derungen globaler Handlungsfelder in Einklang gebracht werden können. Dazu werden abschließend einige Ansatzpunkte für ein interkulturelles Management aufgezeigt.

\section{Inhaltsverzeichnis:}

1. Globalisierung als Managementproblem

2. Zur Kulturproblematik der Globalisierung

3. Organisationales Lernen im interkulturellen Rahmen

4. Ansatzpunktef ür ein Management des interkulturellen Lernens

Literaturverzeichnis 


\section{Globalisierung als Managementproblem}

Globalisierungsprozesse folgen ökonomischen Sachzwängen. Ein solcher ökonomischer Entwicklungsdruck zielt auf die Ausweitung von Wirtschaftsräumen, den schnellen Transfer von Technologie, die kosten- und zeitgünstige Verfügbarkeit von Ressourcen, die Realisierung von Massenproduktionsvorteilen und die Omnipräsenz des Angebots (vgl. Porter 1989; Davis, 1989; Dülfer, 1991). Bei diesem Wettlauf geht es um quantitatives Wachstum. Globaler Wettbewerb erfordert von den einzelnen Kontrahenten, ihre "kritische Masse" ständig zu erhöhen. Allem Anschein nach sind Konzentrationsprozesse und Oligopolisierung eine Konsequenz dieser Entwicklung (Ronen, 1986: 3ff; Prahalad/Doz, 1987: 13ff; Ohmae, 1989: 152f).

Diese Entwicklung wird durch den technischen Fortschritt noch forciert. "A powerful force drives the world toward a converging commonality, and that force is technology. ... The result is a new commercial reality - the emergence of global markets for standardized products on a previously unimagined scale of magnitude." (Levitt, 1983: 92) Obwohl die These von Levitt, daß internationale Unternehmungen nur mit Globalisierungsstrategien überleben können (und mit der Standardisierung der Produkte überall die gleichen Bedürfnisse entstehen, vgl. Levitt, 1983: 100) zunächst der empirischen Überprüfung nicht standhalten konnte (nur 4-8\% aller Produkte sind Standardprodukte (vgl. Krulis-Randa, 1990)), wird jedoch die Globalisierungstendenz deutlich, die aus den veränderten Rahmenbedingungen entstanden ist. Levitts These "nobody is safe from global reach and the irresistable economies of scale" (Levitt, 1983: 102) bringt diese Entwicklung in überspitzter Form auf den Punkt und zeigt recht plastisch den Globalisierungsdruck auf, den Wirtschaftsorganisa tionen in zunehmen dem Ma ße verspüren.

Für das Management resultieren aus dem Globalisierungsszenario zwei Kernprobleme:

- die Bewältigung von Wachstumssprüngen zur Erhöhung der "kritischen Wettbewerbsmasse"

- die Fähigkeit (innere Größe) sich auf globalen Märkten zurechtzu finden.

Das erstgenannte Problem wird gegenwärtig ausgiebig unter den Schlagworten Strategische Allianzen, Unternehmenskooperationen sowie Mergers und Acqusitions behandelt. Das zweite Schwerpunktproblem besteht darin, die Handlungskompetenz von Unternehmen an die Rahmen bedingungen der Globalisierung anzu passen.

Unser Beitrag widmet sich diesem letztgenannten Thema. Uns interessiert also nicht so sehr, wie Unternehmen quantitativ wachsen können und ihre "äußere Größe" erhöhen - 
vielmehr geht es uns um die "innere Größe" und damit um Fragen des qualitativen Wachstums.

Unter qualitativem Wachstum verstehen wir einen Zuwachs an Handlungskompetenz und Problemlösungsfähigkeit, der Unternehmen in die Lage versetzt, in globalen Kontexten zu agieren.

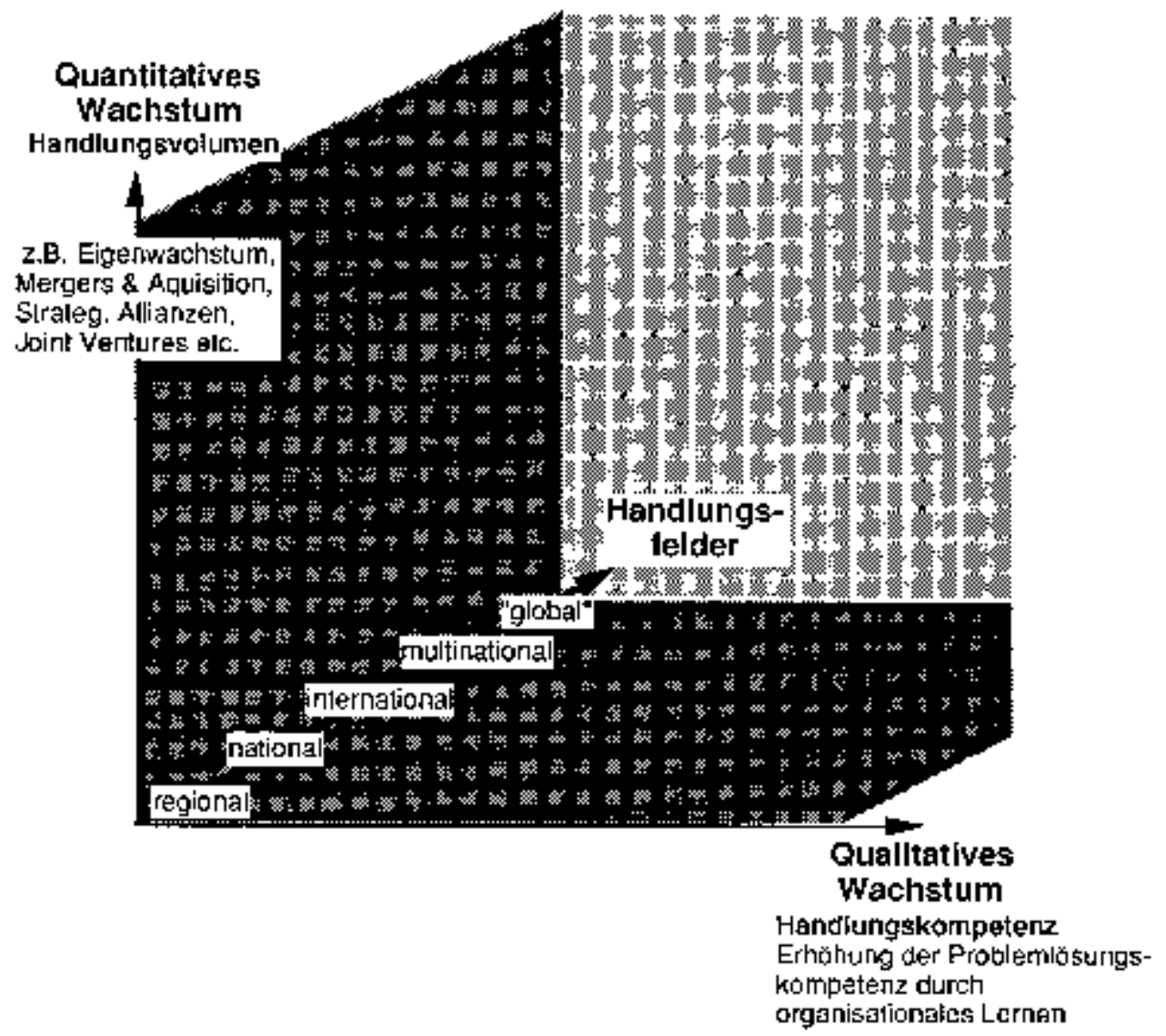

Abb.1: Zusammenhang quantitativen und qualitativen Wachstums

Abb.1: Zusammenhang quantitativen und qualitativen Wachstums

Den Zusammenhang zwischen "innerer" und "äußerer Größe", d.h. zwischen qualitativem und quantitativem Wachstum, skizziert Abbildung 1.

Bilanziert man die bisherige Diskussion zu diesem Thema, so deutet einiges darauf hin, daß Unternehmen ihr quantitatives Wachstum nicht oder nicht zeitgerecht durch ein entsprechendes Wachstum der "inneren Größe" nachvollziehen können. Dies bedeutet, daß die Problemlösungsfähigkeit dem jeweiligen Handlungskontext (international, multinational usw.) nicht hinreichend angepaßt ist. Aus konsistenz- und kontingenztheoretischer Sicht handelt es sich um eine "Fit-Problematik", die zu "Brüchen" in der 
Unternehmensentwicklung führen kann, wenn und so weit es den Unternehmen nicht gelingt, Lernmodelle zu entwickeln, mit denen die Handlungskompetenzen den jeweiligen Handlungsfeldern angepaßt werden (vgl. Abb.2).

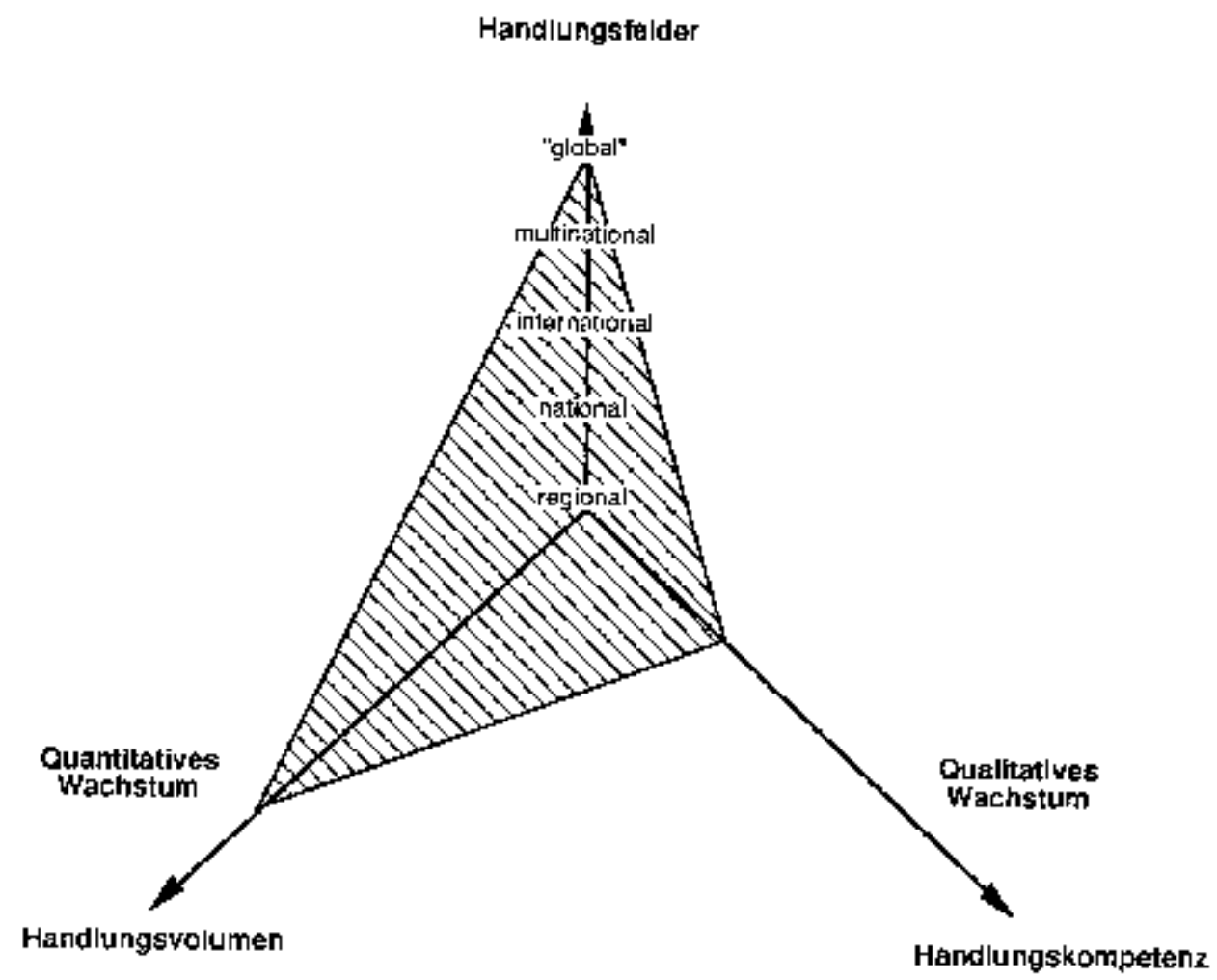

Abb.2 "Frit-Problematik"

Abb.2 "Fit-Problematik"

Unser Vorschlag zur Problembewältigung rückt das "I nterkulturelle Lernen" der gesamten Organisation in den Blickpunkt. Nachfolgend wollen wir deshalb Kultur- und Lernkonzepte zusammenführen 


\section{Zur Kulturproblematik der Globalisierung}

Mit der sich immer schneller vollziehenden technischen sowie medialen Verknüpfung der Wirtschafts- und Gesellschaftssysteme rücken auch deren Kulturräume näher zusammen?. Als $\mathrm{F}$ olge treffen und überschneiden sich unterschiedlichste Kulturräume und leiten damit vielleicht die gegenwärtig so gerne beschworene Epoche einer "multikulturellen Weltgesellschaft" (Hofstede) ${ }^{2}$ ein. Mit der Gesellschaft erfahren auch Wirtschaftsinstitutionen die Auseinandersetzung mit der Kulturproblematik, sind vielleicht sogar deren entscheidender Katalysator. Der Versuch, Probleme und Konflikte zu bewältigen, die aus der Kulturauseinandersetzung entstehen, erzeugt vielfach Verunsicherung, weil liebgewonnene Symbole und Riten an Glaubwürdigkeit verlieren, alte und neue Wertstrukturen aufeinanderprallen und Schattenkulturen ent stehen lassen. 3

Eine aktive Auseinandersetzung mit solchen Veränderungsprozessen kann jedoch auch dazu führen, daß kulturelle Lernfelder entdeckt und zu einer Erweiterung der Handlungskompetenz genutzt werden. Damit entsteht die Chance, interkulturelle Managementtätigkeiten zu einem Wettbewerbsvorteil, und zu einer eigenständigen strategischen Erfolgsposition aus zubauen.

Dies setzt zunächst eine "Sensibilisierung" gegenüber unterschiedlichen und sich verändernden kulturellen Kontexten voraus ${ }^{4}$, die ein Erkennen und reflexives Verarbeiten möglicherweise divergenter Werte und Normen im Rahmen kognitiver und normativer Orientierungsmuster der Organi sation erm öglicht (Schein, 1985).

Interkulturelles Lernen findet im Spannungsfeld zwischen verschiedenen Organisationskulturen unterschiedlicher $\mathrm{Herkunft}^{5}$ (z.B. bei Akquisitionen oder strategischen Allianzen) und/oder direkt zwischen Organisationskultur und fremder Gesellschaftskultur statt (etwa bei Gründung von ausländischen Tochtergesellschaften).

Dieses Aufeinandertreffen und Verschmelzen verschiedener Kulturen zu einem neuen "Gesamtgefüge" eigener Art, kann als Kulturglobalismus" bezeichnet werden.

2 Zitat von Hofstede in seinem Vortrag über "Multikulturelles Management und Weltgesellschaft" auf der 54. Wissenschaftlichen J ahrestagung des Verbandes der Hochullehrer für Betriebswirtschaft am 10.06.1992 in St. Gallen.

3 Der Kulturwandel in Organisationen vollzieht sich herkömmlich durch eine Krise, die Verunsicherung aufwirft und damit Schattenkulturen hervorbringt, die dann zu einem Konflikt zwischen alter und neuer Kultur führt. Wenn die neuen Orientierungen die Krise meistern, so werden die Symbole und Riten dieser Kultur akzeptiert (Steinmann/Schreyögg, 1991: 549).

$4 \quad$ Obgleich diese häufig zitierte Voraussetzung so selbstverständlich und daher kaum erwähnenswert erscheint (vgl. u.a.Hofstede, 1983: 89; Sackmann, 1990: 170; Kumar/Karlshaus, 1992: 60f) bestätigt eine Umfrage unter 500 in Europa tätigen Firmen ihre Bedeutung. Die Studie zeigt, daß nur $11 \%$ der Firmen den gekonnten Umgang mit nationalen Kulturen als ein adäquates Mittel ansehen, auf kulturelle Unterschiede reagieren zu können und nur $12 \%$ die Anpassungsfähigkeit an unterschiedliche Kulturräume als ein wichtiges Merkmal einer paneuropäischen Corporate Identity betrachten (vgl. Schmidt, 1991: 61ff).

Wobei zu beachten ist, daß Organisationkulturen zwar auf den sie umgebenden Gesellschaftskulturen basieren, mit diesen jedoch nicht identisch sind (vgl. z.B. den Beitrag von Schreyögg in diesem Band). 


\section{Kultur als Interpretationsmuster}

(Organisations-)Kultur ist ein im Rahmen von Lernprozesen erworbenes Wissens- und Erkenntnissystem, das der Interpretation von Erlebnissen (Erfahrungswissen) und der Genese von Handl ungen (Aktionswissen) dient (Klimecki/Probst, 1990).

Kultur ist somit ein "implizites Phänomen", das in gemeinsamen Werten und Orientierungen zum Ausdruck kommt (Steinmann/Schreyögg, 1991). "Kultur entsteht, geschieht und verändert sich interaktiv, durch Wechselwirkungen und Austauschbeziehungen innerhalb des Systems und gegenüber Umwelteinflüssen" (Klimecki/Probst, 1990: 41).

Kultur manifestiert sich in verschiedenen gesellschaftlichen und organisationalen Bereichen - z.B. in Objekten (einheitliche Kleidung), Sprache (Geschichten, Legenden usw.), in Verhalten (Routinen, Bräuche) sowie in Gefühlen (Sicherheit, Gleichbehandlung usw.) (vgl. Sathe, 1983: 17f). Diese unterschiedlichen Manifestationen können verschiedenen Ebenen zugeordnet werden. ${ }^{6}$ Aus den internen sowie externen Interaktionen der Organisation entstehen Erfahrungsmuster, die sowohl auf der materiellen wie auf der geistig-sinnhaften oder symbolischen Ebene repräsentiert sind. Nicht die "materiellen Tatsachen", sondern deren Wahrnehmung und Interpretation erzeugen Kultur. Daraus folgt, daß Kultur nicht als etwas aktiv und direkt Steuerbares begriffen werden kann, sondern vielmehr als Entwicklungsprozess, in dem normative Ordnungsmuster durch ein interaktives Zusammenspiel der Teile zu einem Ganzen zusammenfügt werden (vgl. Schein,1989: 58ff). "Zusammengefaßt ist Kultur damit eine Sichtweise für das ganze System - und in diesem Sinne ein Metakonzept zur Beschreibung, Erklärung und Gestaltung einer Ganzheit." (Klimecki/Probst, 1990: 43)

\section{Interkulturelle Lernfelder und Konfliktpotentiale}

Ausrichtung und Inhalt interkultureller Lernmodelle sind stark davon abhängig, welche Annahmen über die zuvor genannten Differenzen zwischen Gesellschafts- bzw. Organisationskulturen und die ihnen möglicherweise innewohnenden Konfliktpotentiale getroffen werden.

Zur Systematisierung dieses Problems kann etwa von folgenden Einflußebenen ausgegangen werden:

- der Einflu ß der ökonomischen Sphäre auf die Kulturebene,

- die Kulturabh ängigkeit des Managements,

Der von Schein (1985) aufgezeigte Weg einer dreistufigen Differenzierung in Artefakte, Werte/Normen und basale Annahmen, auf den später noch näher eingegangen wird, zeigt jedoch zugleich auch die Schwierigkeiten der methodischen Erfassung auf, der vor allem daraus resultiert, daß der eigentliche kulturelle Kern weitgehend in den "Tiefenlagen" des kollektiven (Unter-)Bewußtseins verborgen ist. 
- die Wechselwirkungen zwischen Unternehmens- bzw. Managementkulturen und Gesellschaftskulturen.

\section{Die ökonomische Prägung von Gesellschaftskulturen:}

Aus naheliegenden Gründen wollen wir uns auf eine Vertiefung dieses Bereichs nicht weiter einlassen. Einige Anmerkungen sind jedoch zum Verständnis der nachfolgenden Aspekte von Bedeutung. Es steht wohl außer Zweifel, daß die Bedingungen des "Wirtschaftens" auf die Entwicklung gemeinsam geteilter Norm- und Wertvorstellungen auch geschichtlich gesehen einen großen Einfluß genommen haben. Kulturen, die über eine große wirtschaftliche Potenz verfügten, haben es schon immer verstanden, sich durchzusetzen, und andere wirtschaftlich weniger erfolgreiche Kulturen zu verdrängen. Es ist deshalb wohl naheliegend, Wirtschaft als einen wichtigen kulturellen Katalysator (und "Transporteur") anzusehen. Diese, in ihrer Grundaussage wohl allgemein geteilte Erkenntnis bildet jedoch nur den Hintergrund dafür, ob es eine bestimmte Art von - wie wir es nennen - "innerer Größe", also von Handlungskompetenz gibt, die sich quasi übersituativ, d.h. in allen denkbaren kulturellen Handlungskontexten als gleichermaßen erfolgreich erweist und damit große Chancen hat, sich durchzusetzen und wiederum kulturprägend zu werden. Dies leitet zur zweiten Frage über, inwieweit Management als kulturgebundenes Element anzusehen ist. 
Kulturgebundenheit von Management:

Bekanntlich stehen sich hier im wesentlichen zwei Thesen gegenüber, aus denen sich im Verlauf der wissenschaftlichen Diskussion verschiedene "Mischtypen" ergeben haben. Im wesentlichen geht es um das Problem, ob Management als kulturgebunden oder kulturfrei anzusehen ist. Auch diese Frage wollen wir hier nicht ausführlich vertiefen. Allerdings gehen wir von bestimmten Grundannahmen aus, die nicht ohne Auswirkungen auf unser interkulturelles Lernmodell bleiben. Wir halten Management für ein kulturgebundenes Phänomen, ${ }^{8}$ das gleichwohl Elemente aufweist, die sich interkulturell als relativ stabil erweisen dürften. Diese stabilen Elemente liegen sowohl in der instrumentellen und/oder operativen als auch in der normativen Dimension des Managements. So finden wir auf der instrumentellen und operativen Ebene durchaus Planungs- und Kontrollverfahren, Methoden der Arbeitsorganisation und der Gestaltung von Führungsprozessen, die in unterschiedlichen kulturellen Kontexten sehr ähnlich ausgestaltet sind und deshalb (zumindest auf den ersten Blick) ein hohes Maß an Stabilität nachweisen (vgl. Hofstede, 1980). Auf der normativen Ebene läßt sich ebenfalls aufzeigen, daß bestimmte Werte und Ziele des Managements nicht als kulturspezifisches Allgemeingut anzusehen sind oder sich als solches durchgesetzt haben (z.B. Leistung als Wert, Produktivität als Megaziel, das ökonomische Prinzip als Entscheidungsformel). ${ }^{9}$ Diese kulturunspezifischen Artefakte (z.B. Instrumente) und Werte werden jedoch u.U. kulturunterschiedlich angewendet bzw. bedeuten auch Unterschiedliches. Managementhandeln kann deshalb nicht unabhängig vom kulturellen Kontext gesehen werden, weil dieser das Deutungsmuster liefert für die Erfassung, Bewertung und Interpretation des Managerhandelns (vgl. Klimecki/Probst/E berl, 1991: 147). Somit können gleiche Verfahren unterschiedlich angewendet und interpretiert werden. Und gleichartig anmutende Wert- und Zielvorstellungen besitzen in unterschiedlichen Kulturen auch einen unterschiedlichen Deutungsgehalt, d.h. sie werden unterschiedlich operationalisiert und "rangiert". So macht es wohl einen Unterschied, ob "Leistung" individuell oder kollektiv verstanden wird und ob sie z.B. in "kleinen Schritten" oder "großen Sprüngen" zum Ausdruck kommen soll.

Hier sei auf die verschiedenen Ansätze der Managementforschung des "Comparitive Management", und die Diskussion zwischen den sog. Kulturalisten ("culture-bound-thesis") mit den sog. Universalisten ("culture-freethesis") verwiesen (vgl. u.a. N. Adler, 1983, S.29ff; Negandhi, 1983: 22ff; Ronen, 1986; N. Kumar, 1988, S.389ff).

Vgl. Habermas: "Mangementaspekte, die auf Normen und persönlichen Erlebnissen der Aktoren beruhen, werden ... von kulturgebundenen Geltungsansprüchen begleitet." (1982: 394)

9 Man muß sich jedoch bewußt sein, daß dies nicht für alle uns bekannten Gesellschafts-/ Ethnokulturen gilt. In historischer Betrachtung ist diese Auffassung deshalb u.U. tautologisch, da Management dieser Art eben ein spezifisches Kennzeichen von Kulturen mit hoher wirtschaftlicher Potenz ist, d.h. dieses Managementverständnis ist in den Kulturen zu finden, die sich durchgesetzt haben. Die Kulturinvarianz des Managements ist nach dieser Auffassung nichts anderes als das Ergebnis eines Verdrängungsprozesses und damit eine Art "sozialdarwinistischer self-fulfilling prophecy". 
Dies bedeutet, daß an sich kulturunspezifisch wirkende Elemente des Managements doch erst dann verständlich werden und ihren "Sinn offenbaren", wenn man sich ihnen mit kulturspezifischen Interpretationsschemata nähert. Aus dieser Perspektive werden die Wechselwirkungen zwischen Unternehmens-, (bzw. allgemeiner) Managementkulturen und Gesellschaftskulturen besonders offensichtlich. Daß es wenig realistisch ist, von einem konfliktfreien Verhältnis auszugehen, sondern durchaus Konkurrenz und "Verdrängungswettbewerb" bestehen kann, wollen wir mit den nachfolgenden Beispielen skizzieren.

Wechsel wirkungen zwischen Unternehmens-, bzw. Management- und Gesellschaftskultur: 11

Aus unseren bisherigen Ausführungen läßt sich ableiten, daß Management als kulturgebundenes Phänomen zugleich auch Träger der jeweiligen Umgebungskultur (vgl. Abb. 3) ist. Im Zuge der Globalisierung haben sich somit die Managementkulturen global agierender Unternehmen mit anderen Umgebungskulturen auseinanderzusetzen.

Im Spannungsfeld der Begegnung verschiedenartiger Management- und Gesel Ischaftskulturen lassen sich verschiedene Konfliktpotentiale $\quad{ }^{12}$ auf zeigen.

- Wenn wir davon ausgehen, daß Unternehmens- bzw. Managementkulturen immer auch ein Produkt der Gesellschaftskultur sind, begegnen sich auf globalen Handlungsfeldern damit nicht nur die Managementkultur eines Unternehmens mit den Gesellschaftskulturen der jeweiligen "Gastländer", sondern zugleich transportiert die Manage mentkultur auch die Gesellschaftskultur des Herkunftslandes in einen

11 Für die folgenden Ausführungen scheint uns der Begriff "Managementkultur" treffender als der Begriff Unternehmens- oder Organisationskultur, da mit letzterem üblicherweise ein "Unikat" (d.h. die spezifische Kultur eines Unternehmens) assoziiert wird. Zugleich verdeutlicht der u.E. allgemeinere Begriff Managementkultur auch besser, worauf sich die potentiell konfliktgeladenen Wechselwirkungen beziehen: Auf Divergenzen und Widersprüche zwischen den Wertstrukturen eines Unternehmens und denen eines Gastlandes oder eines ausländischen Kooperationspartners, die durch konfliktäre Managementhandlungen symbolisiert werden. 


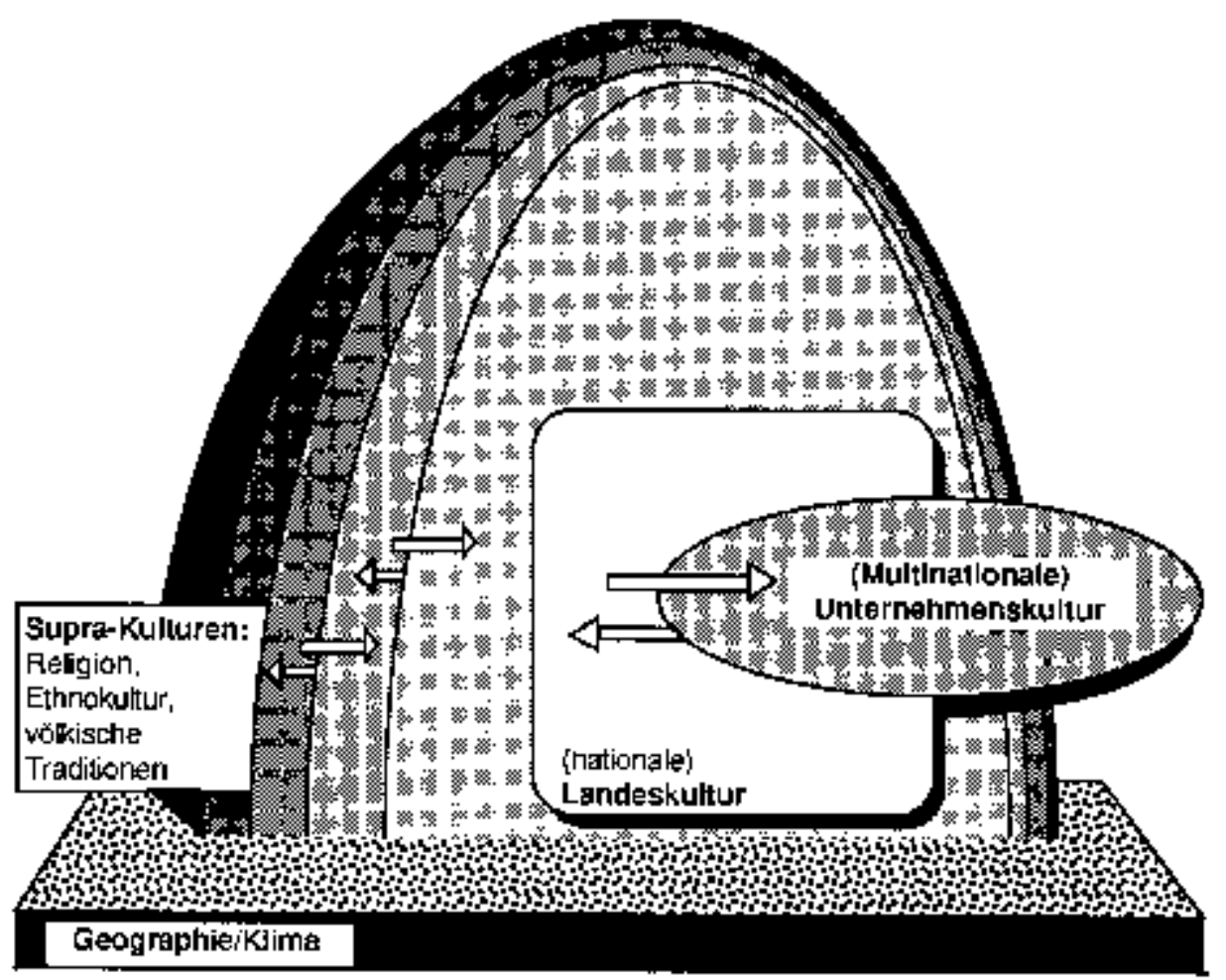

Abb.3 Schichtereradell der Kutturien ils

Abb.3 Schichtenmodel der Kultur/ en $\quad 13$

globalen Kontext. Mögliche Auseinandersetzungen zwischen Management- und Gesellschaftskulturen sind deshalb nur verständlich, wenn die Basiskultur der jeweiligen Herkunftsländer miteinbezogen wird. Somit konkurrieren z.B. nicht nur die Managementkulturen japanischer und deutscher Unternehmen, sondern zugleich auch immer die jeweils dahinterstehenden Gesellschaftskulturen. Dies kann zum einen den bereits angesprochenen aggressiven Verdrängungswettbewerb forcieren (die z.B. gelegentlich in der "japanischen Bedrohung" zum Ausdruck zu kommen scheint (vgl. Vahlefeld, 1992)). Andererseits könnte aber hier eine sich unter dem Druck globalisierender Wettbewerbsbedingungen notwendigerweise kosmopolitisch orientierende Managementkultur auch als Vermittler zwischen divergierenden Nationalkulturen etablieren.

Die hier möglicherweise entstehenden Widersprüche und Effekte lassen sich z.B. wie folgt verdeutlichen:

a) "Kultur-Globalismus": Durch Globalisierung und Internationalisierung entstehen Kulturen "eigener Art" - d.h. spezielle Formen von Managementkulturen, die nicht mehr auf ihre ursprünglichen Gesellschaftskulturen zurückführbar sind. Sie liegen 
vielmehr "quer" zu den jeweiligen Landeskulturen, in denen multinationale Unternehmen domizilieren. Viele dieser Managementkulturen dürften aufgrund der dahinterstehenden Wirtschaftskraft "mächtiger" sein als die sie umgebende Gesellschaftskultur.

b) E in weiteres Problemfeld sind die "Kulturschizophrenien", die durch ein gleichzeitiges Erleben widersprüchlicher Wertsysteme (Gesell- schaftskulturen vs. globale Managementkulturen) entstehen können und denen immer mehr Managerinnen und Manager immer inten siver ausge setzt sein d ürften (vgl. Schneider, 1988: 241ff).

c) Ein drittes Beispiel wäre "Kulturarroganz", etwa in Form einer rücksichtslosen Änderung gewachsener Wertstrukturen, z.B. bei feind lichen Übernahmen.

- Ein weiteres Konfliktfeld kann dadurch entstehen, daß, wie gelegentlich aufgezeigt wird, die Gesellschaftskultur gegenüber der Managementkultur durch ihren stärkeren Einfluß auf die Annahmen und Einstellungen der Managerinnen und Manager grundsätzlich dominanter wirkt (vgl. Laurent, 1986: 93). So vertreten z.B. Wilkins und Ouchi (1983) die Auffassung, daß Unternehmenskulturen weniger tief als ihre Umgebungskulturen verankert sind, und daß es daher leichter fallen dürfte, als allgemein angenommen, Unternehmenskulturen zu verändern. Trifft dies zu, dann lassen sich zwei mögliche Effekte ableiten:

Zum einen wäre dann die Managementkultur tatsächlich ein potentieller Harmonisator, weil sie "anschmiegsamer" als die Umgebungskulturen ist. Zum anderen besteht aber die Gefahr, daß sie deshalb anfälliger für konkurrierende Sub- und Schattenkulturen ist. Damit würden globale Managementkulturen möglicherweise viel zu häufig Konfliktsituationen ausgesetzt sein, um ihrer Integrations- und Identifikationsaufgabe noch gerecht werden zu können - gerade dann, wenn es darauf ankommt, d.h. in grundsätzlichen (tiefgreifenden) Streß- und Konfliktsituationen.

Die jetzt besonders notwendigen tiefgreifenden Lernvorgänge würden in diesem Fall durch "defensive routines" (Argyris, 1990) der miteinander in Konflikt stehenden "Schattenkulturen" vermutlich verhindert.

- Auch stellt sich die Frage, welche Auswirkungen diese Kulturphänomene auf das Handeln von Managerinnen und Managern nehmen können. Die These, daß Umgebungskulturen stärker sind als Managementkulturen, wird auch durch die Managerforschung unterstützt. Hier zeigen Ergebnisse auf, daß Managerinnen und Manager vor allem in Streßsituationen in gesellschaftskulturelle Verhaltensmuster zurückfallen (vgl. Literatur aus der Psychologie, wie u.a. Brislin/Bochner/ Lonner, 1975; Brislin/Cushner/Cherrie/Young, 1985). 
Im Zweifel ist ein japanischer Manager also in erster Linie Japaner und dann erst Manager einer "multikulturell beeinflußten" Unternehmung. Sollten also interkulturelle Konflikte auftreten und keine geeigneten organisationalen Lern- bzw. Konfliktbewältigungsmodelle zur Verfügung stehen, ist nicht zu erwarten, daß sich die Probleme auf der Ebene des individuellen Lernens und Verhaltens Iösen Iassen. Dies führt zu der U.E. wichtigen Hypothese, daß der vielbeschworene "just-one-worldmanager" wohl nur dann Erlebens- und Überlebensmöglichkeiten hat, wenn er in einer "just-one-world-company" tätig ist.

In der gegenwärtigen Diskussion wird jedoch die entgegengesetzte These vertreten, d.h. interkulturelle Probleme würden sich durch eine genügende Anzahl von "just-one-worldmanagern" von sel bst lösen. $\quad 15$ 


\section{Organisationales Lernen im interkulturellen Rahmen}

Um interkulturelles Lernen als eine Möglichkeit der Bewältigung von Globalisierungsprozessen näher zu beleuchten, empfiehlt es sich, von Konzepten des organisationalen Lernens auszugehen und diese mit Ansätzen zur Unternehmenskultur bzw. zum kulturbezogenen Mana gement in Verbindung zu bringen.

Nach den bisherigen Ausführungen scheint uns die übliche Auffassung, nach der Unternehmenskulturen global tätiger Unternehmen durch verschiedenste nationale Umgebungskulturen beeinflußt werden und zu einer "Multikultur" des Unternehmens verschmelzen ${ }^{16}$, zu stark vereinfachend oder doch zumindest zu harmoniegläubig (vgl. Negandhi/Prasad, 1970; Hofstede, 1980; Bartlett/Goshal, 1989). Ebenso gehen wir davon aus, daß entgegen der von Schreyögg (in diesem Band) zumindest implizit vertretenen Auffassung, keine "Wahlfreiheit" zwischen globalen und ethnozentrischen Unternehmenskulturen besteht. Der Start zu einem multinationalen oder globalen Unternehmen beginnt zunächst auf der Basis einer stark von der Heimatkultur geprägten Unternehmenskultur. Da diese dem Unternehmen seine Identität verleiht und einen Speicher für die Erfolgsgeschichten und den Stolz der Organisation darstellt, dürfte sie auch ein gewisses Beharrungsvermögen aufweisen (vgl. Klimecki/Probst, 1990). Je größer die kulturellen Sprünge sind, die bei der Globalisierung überwunden werden müssen, desto mehr steigt auch die Gefahr, daß die in einer neuen "Multikultur" zu integrierenden Werte auf Unverständnis stoßen, nicht entschlüsselt werden können oder sogar mit der Unternehmenskultur in Konflikt geraten. Die Gefahr neuartiger Konflikte dürfte dabei umso größer sein, je stärker die konkurrierenden neuen Werte die alte Identität des Unternehmens gefährden. Es zeigt sich, daß interkulturelles Lernen in der gesamten Organisation und durch die Organisation stattfinden muß. Hierbei ist das Lernen der auslandsentsendeten Manager von ihrem kulturellen Umfeld zweifelsohne eine wichtige aber keinesfalls hinreichende Voraussetzung zur Bewältigung von Kulturproblemen der Globalisierung.

Dieser "Verschmelzung" liegt häufig die Vorstellung von Unternehmenskultur und Ethnokultur als monolithische Blöcke zugrunde. Eine zeitlich dynamische Betrachtungsweise zeigt jedoch, daß im Laufe der Zeit monolithische Kulturen in Subkulturen zerfallen (vgl. Kasper, 1987: 37ff; Kiechl, 1990: 120). 


\section{Zum Konzept des organisationalen Lernens}

Konzepte des organisationalen Lernens argumentieren in der Regel metaphorisch, d.h. sie übertragen den individualistisch geprägten Begriff des Lernens analog auf "überindividuelle Ganzheiten". Organisationales Lernen beschreibt dabei ein auf individuelles Lernen zurückgehendes aber nicht mit diesem gleichzusetzendes (bzw. linear darauf zurückführbares) Phänomen. 17

Damit werden zwei Prämissen zugrunde gel egt:

a) Organisationales Lernen erfolgt über individuelles Lernen (die "lernenden Subjekte" sind I ndividuen) und

b) Organisationen können Wissen unabhängig von ihren Mitgliedern in "kognitiven Landkarten" (wie Hedberg es bezeichnet) speichern. In diesen "cognitive maps" wird jedoch nicht das gesamte individuelle Wissen "eingraviert" sondern nur jene Bestandteile, die für die Organisation nützlich und akzeptabel sind (vgl. Hedberg, 1981; Weick, 1986). 18

Mit den Selektionskriterien Nützlichkeit und Akzeptanz sind zwei vorherrschende Definitionen von organisational em Lernen angesprochen:

a) Die Organisation lernt, wenn sie ihre Wissensbestände erhöht (Duncan/Weiss, 1979). Sie speichert Wissen über $\mathrm{H}$ andlungsstrategien und Handlungsfolgen. Hier ist vor allem die Frage der Nütz lichkeit angesprochen.

b) Die Organisation lernt, wenn sich ihre Grundannahmen bzw. Werte (Kultur) in einer Weise verändern, daß neue (und bessere) Handlungskriterien erzeugt werden (Argyris/Schön, 1978). Hier ist insbesondere die Dimension der Akzeptanz entscheidend.

Wir wollen beide Ansichten von organisationalem Lernen miteinander verbinden, da wir zwischen ihnen eine offenkundige Wechselwirkung sehen. Diese besteht einerseits darin, daß erfolgreiche Handlungsweisen über die Zeit hinweg normierend wirken und über Rituale in einen kulturellen Status überführt werden können. Andererseits lassen sich gemeinsam geteilte Grundannahmen (also institutionell verfestigte Wertmuster) auch als Selektionsmechanismen für die Akzeptanz von Handlungsstrategien und die damit verbundene Informationsauswahl betrachten. Sie entscheiden z.B. darüber, welche Erfol gsgeschichten als solche anerkannt werden und wie sie zu kommunizieren sind.

Vgl. die Zusammenstellung der verschiedenen Theorien organisationalen Lernens: Shrivastava, 1983; Fiol/Lyles, 1985; Levitt/March, 1988)

18 Daraus folgt, daß Organisationen zugleich sowohl mehr (Argyris/Schön 1978; Fiol/Lyles 1985) als auch weniger (Hedberg 1981: 6) "wissen" als ihre Mitglieder. Organisationales Wissen unterscheidet sich somit vom individuellen, weil es eine "andere Qualität" besitzt.

19 Die nachfolgende kleine Geschichte mag ein Beispiel für diese Wechselwirkung geben. Es wird kolportiert, daß in 
Die Grundvoraussetzung für ein organisationales Lernen besteht deshalb in "Veränderungen der organisationalen Wert- und Wissensbasis". Diese Veränderungen sind dann mit einer Höherentwicklung, also mit einem Zugewinn an innerer Größe und qualitativem Wachstum verbunden, wenn sie zu einer Erhöhung der Handlungskompetenz und Problemlösungs fähigkeit der Organisation führen wird.

\section{Ein Orientierungsmodell zum interkulturellen organisationalen Lernen}

Kulturbezug und organisationales Lernen sind die Schlüsselgrößen für qualitatives Wachstum. Ein für die Bewältigung von Globalisierungsprozessen notwendiges Maß an "innerer Größe" erfordert deshalb Entwicklungsprozesse, die auch "auf Dauer" angelegt sind und deren Ziel es ist, einen Gleichklang zwischen Wissensbasis sowie Wertstrukturen der gesamten Organisation und den Anforderungen globaler Aktionsfel der zu erzeugen.

Bevor Empfehlungen für die Ausgestaltung solcher Entwicklungsprozesse entworfen werden können, ist es zunächst notwendig, ein gemeinsames konzeptionelles Bild zu finden, mit dem die Wechselbeziehungen von Kultur und organisationalem Lernen aufgezeigt werden können. Ein solches Bild kann durch die Integration von Kultur- und Lernkonzepten gewonnen werden. Dabei sind Ansätze, die (zumindest implizit) bereits aufeinander verweisen, für dieses "Orientierungmodell interkulturellen organisationalen Lernens" aus naheliegenden Gründen besonders geeignet (Abb.4).

Ein Kulturkonzept, das (organisationale) Lernphänomene in den Mittelpunkt rückt, wurde von Schein (1985) vorgeschlagen: Kultur entsteht in einem komplexen Wechselspiel von externen Zwängen, internen Potentialen, Reaktionen auf kritische Ereignisse sowie durch Einwirkungen nicht vorhersehbarer Veränderungsfaktoren und wird verstanden als "a pattern of basic assumptions - invested, discovered or developed by a given group as it learns to cope with its problems of external adaptation and internal integration." (ders. S. 9) Kultur ist damit " ... the outcome of group learning. When a number of people simultaneously face a problematic situation and have to work out a solution together, we have the basic situation for culture formation." (ders. S. 183)

der Walt Disney Corporation jede wichtige Entscheidung vor ihrer endgültigen Ratifizierung noch einmal auf einen ganz besonderen Prüfstand gestellt wird. Das Management fragt sich nämlich, "Was würde Walt (Disney) an unserer Stelle getan haben?" 


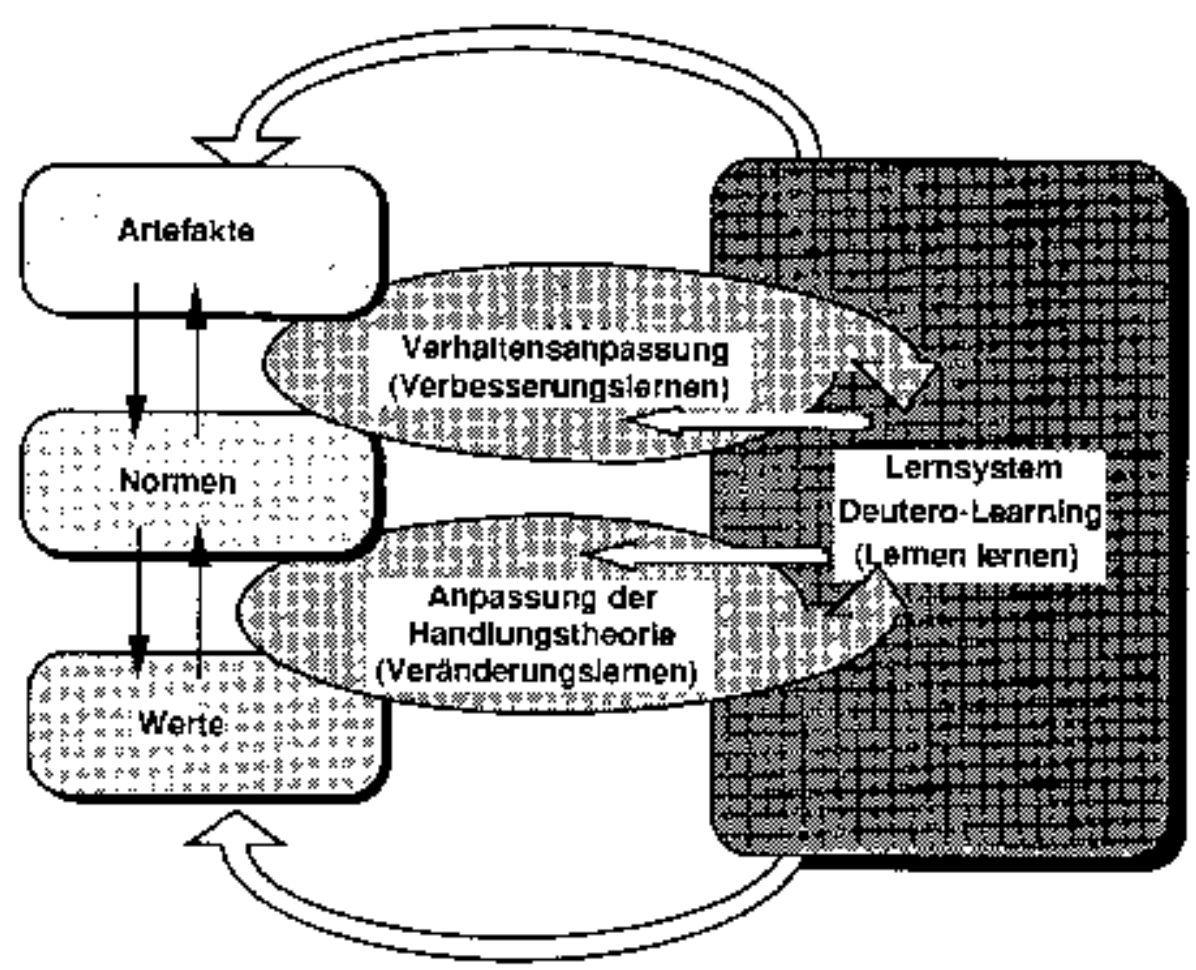

Abb.4: Orientierung:madell eines kulturbezogenen $\gamma_{\text {ernens }}{ }^{20}$

Abb.4: Orientierungsmodell eines kulturbezogenen Lernens

Dieser Auffassung, Kultur als jenes Muster gemeinsam geteilter Grundannahmen anzusehen "... that has worked well enough to be considered valid and therefore, to be taught to new members, as the correct way to perceive, think, and feel in relation to those problems" (Schein, 1985: 9), würden Argyris/Schön (1978) in ihrem Konzept des "organizational learning" wohl kaum widersprechen. Sie bezeichnen organisationales Lernen als eine Veränderung "geteilter Grundannahmen", die tiefgreifend genug ist, neue und andersartige $\mathrm{H}$ andlungstheorien hervorzubringen (dies., 1978: 10). Betont wird damit der dynamische Aspekt der Kultur: Organisationen lernen (d.h. "verbessern" ihre Handlungskompetenz), wenn und soweit sie in der Lage sind, ihre Kultur zu transformieren. Betrachtet werden damit jene Situationen, in denen die vorherrschenden "basic assumptions" nicht mehr in der Lage sind, Handlungstheorien hervorzubringen, mit denen die Probleme der externen Anpassung und der internen Integration gelöst werden können. Der von einer solchen Kultur gewiesene Weg, wahrzunehmen, zu denken und zu fühlen ist nicht mehr "korrekt", weil er eine adäquate Problembewältigung verhindert. 
In Anlehnung an die von Bateson (1981) vorgenommene Differenzierung von "first" und "second order learning" unterscheiden Argyris/Schön (1978) drei Lernniveaus:

- "Single loop learning" oder "Verbesserungslernen", welches in einer Verfeinerung der bisherigen $\mathrm{H}$ andlungstheorie besteht, keine Kulturtransformation erfordert und das deshalb kein eigentliches organisatio nales Lernen darstellt.

- "Double loop learning" oder "Veränderungslernen", welches neue, andersartige $\mathrm{H}$ andlungstheorien entstehen Iäßt und somit nur über Kulturtransformation zu erreichen ist.

- "Deutero learning", d.h. die F ähigkeit, Lernprozesse zu reflektieren und zu verbessern.

In seinem Kulturkonzept unterscheidet Schein ebenfalls drei Stufen, die Auskunft über die "Tiefenstruktur" der Kultur geben sollen:

- Artefakte; die sichtbarste und am leichtesten zu erschließende Kulturebene, auf der "basic assumptions" durch Symbole "materialisiert" werden.

- Normen; nachvollziehbare und diskussionsfähige Vorstellungen über gewünschte Sollzustände.

- Werte bzw. basale Annahmen; d.h. unter- oder unbewußte Leitbilder, die nur schwer erschlossen werden können.

Veränderungslernen (double loop learning) erfolgt duch die Transformation von Werten und Normen. Dies bedeutet eine Veränderung der kulturrellen Tiefenstruktur und kann nur dann erfolgen, wenn es gelingt, die teilweise verborgenen und eng mit der Identität (von Person und Organisation) verbundenen Grundannahmen, Welt- und Leitbilder offenzulegen und einer "kritischen Prüfung" im Sinne eines kulturbezogenen Reflexionsprozesses zu unterziehen. Dies ist deshalb besonders schwierig, weil die auf dieser Tiefenstruktur basierenden Handlungstheorien ("theories in use", wie Argyris/Schön sie bezeichnen) von den offiziell kommunizierten "Bekenntnistheorien" nicht selten abweichen und ihre kritische Reflexion tabuisiert wird. Organisationen bauen "defensive routines" (Argyris 1990) auf, die sie vor den Verunsicherungen schützen sollen, die unabdingbar mit organisationalem Lernen und dem damit möglicherweise verbundenen "Kulturschock" einhergehen. Bestehende "basic assumptions" werden deshalb durch fundamentale Barrieren geschützt, die zu einer Ignoranz von Fehlern führen, die Diskussion dieser Fehler verbieten und auch über deren "Nicht-Diskutierbarkeit" nicht diskutieren lassen.

Single und double loop learning entspricht dem "first order learning" bei Bateson, welches als instrumentelles Lernen auf Objekte gerichtet ist, die außerhalb des Lernprozesses selbst liegen (also z.B. Lernen, eine neue Marketingstrategie zu entwickeln). Beim "second order learning" ist hingegen das Lernen selbst der Lerngegenstand (Lernen, wie man lernt). 
Aus dem hier skizzierten kulturbezogenen Lernmodell Iassen sich verschiedene Schlußfolgerungen für die Analyse und Gestaltung interkultureller Lern- bzw. Konfliktprozesse ableiten. Die wichtigsten Konsequenzen sollen nachfolgend aufgezeigt werden:

- Kultur entwickelt sich im Zuge von Organisations-Umwelt-Interaktionen. Da sich die Umweltbezüge der Organisation durch die Globalisierung stark verändern, ist davon auszugehen, daß die ursprünglichen "basic assumptions" (bzw. die darin verfestigten Handlungstheorien) zur Lösung des sich in diesem neuen Kontext auch wieder neu stellenden Problems der "externen Adaptation" nicht ausreichen. Die Unternehmenskultur läuft somit Gefahr "falsch" zu werden, weil sie die Organisationsmitglieder "unkorrekt" orientiert. Für das Handlungsfeld, auf dem agiert wird, ist man nicht (mehr) "kompetent". Es entstehen Diskrepanzen zwischen "äußerer" und "innerer Größe"; das quantitative Wachstum vol lzieht sich schneller als das qualitative.

- Bei zunehmender Präsenz auf globalen Märkten wird auch der Veränderungsdruck im System zunehmen, da sich über die Kooptation ausländischer Mitarbeiter sowie über die steigende Anzahl auslandserfahrener Mitglieder aus den eigenen Reihen das interne Potential (als eine weitere kulturbildende Einflußgröße) verändert. Auch die zweite organisationale Hauptaufgabe, die interne Integration, stellt sich also neu und läßt sich vermutlich nur bewältigen, wenn Veränderungslernen statt $\quad$ findet.

- Durch fortdauernde globale Präsenz wird die eigene Unternehmenskultur ständig von Werten und Normen fremder Unternehmens- und/oder Landeskulturen "infiltriert". Dies begünstigt "Kulturschizophrenien" und kann auch zu "Kulturarroganz" führen (entsprechende "defensive routi nes" und hinreichende Wirtschaftsmacht vorausgesetzt).

- Interkulturelle Konfliktpotentiale nehmen zu, je breiter der "kulturelle Korridor" (Macharzina) ist, und je weiter er in die Tiefenstruktur der beteiligten Kulturen hineinragt. Diese These klingt zunächst trivial, ist jedoch sehr praxisrelevant. Sie bedeutet, daß interkulturelles Management umso komplizierter wird, je mehr Unterschiede auf einer Kultur-ebene vorzufinden sind - und sie bedeutet auch, daß interkulturelles Management umso komplexer wird, je "tiefer" diese Unterschiede liegen.

- Aus Lage und Umfang kultureller Differenzen (also aus der Breite und Tiefe des Korridors) Iassen sich Ansatzpunkte für organisationale Lernstrategien ableiten: Die mit "Verbesserungslernen" (single loop learning) erzeugbare zusätzliche Handlungskompetenz dürfte nur für Kulturdifferenzen auf der Ebene der Artefakte und 
im Bereich leicht erschließbarer, wenig konfliktärer Normen ausreichend sein. Beziehen sich die Kulturdifferenzen auf widersprüchliche Normen und Basiswerte, müssen neue Handlungstheorien erzeugt werden - ein "Veränderungslernen" (und damit eine Transformation der basalen Annahmen) wird unum gänglich.

- In jedem Fall gilt es jedoch, zunächst einmal zu erkennen, welche Art von Kulturdifferenz zu überbrücken ist. Auswahl und Gestaltung der "richtigen" Lernstrategie hängen somit primär von der Fähigkeit zum "deutero learning" ab. Solche "Lernvorgänge der zweiten Ordnung" können etwa die folgenden wichtigen Funktionen erfüllen:

- Reflektieren der Lernsituation und Auffinden interkultureller Lernfelder bzw. Sichtbarmachen von Konflikten und "kulturellen Unzu Iänglichkeiten"

- Erkennen von Widerspr üchen zwischen Bekenntnis- und Hand lungs theorien

- Aufdecken von Defensivroutinen

- Ermitteln dessen, was "verlernt" werden mu B B (Hedberg,1981)

- Institutionalisierung lernfreundlicher Strukturformen

- Anregung zur interkulturellen Kommunikation 


\section{Beispiele zur Illustration des Orientierungsmodells}

Die mit Hilfe unseres Orientierungsmodells zunächst abstrakt aufgezeigten Problemfelder wollen wir mit einigen Beispielen verdeutlichen.

Unser erstes Beispiel beschäftigt sich mit Unterschieden auf der Ebene der Kultursymbole (Artefakte).

Eine U mfrage über die Zusammenarbeit zwischen Deutschen und Franzosen (J PB-Studie) ${ }^{22}$ zeigte Differenzen auf, die weitgehend der "Kulturoberfläche" zuzurechnen sind. ${ }^{23}$ So gab etwa die Hälfte der befragten Mitarbeiter deutsch-französischer Unternehmen an, daß die Kooperation mit den jeweils anderen "Kulturvertretern" von Problemen belastet wird, die auf "M entalitätsunterschiede" (unterschiedliche Symbole und Normen) zurückzuführen sind. Diese äußern sich in Mißverständnissen, z.B. aufgrund unterschiedlicher Arbeitsweisen. Dabei waren Äußerlichkeiten wie Sprache und Verhalten der vorwiegende Grund für Auseinandersetzungen. So sind etwa die deutschen Mitarbeiter gewohnt, in der Regel nur einen Arbeitsvorgang auf einmal zu verrichten, während die Franzosen dazu neigen, mehrere Vorgänge gleichzeitig abzuwickeln. Als weitere Probleme wurden unterschiedliches Hierarchieverständnis, ineffiziente Konferenzen und Besprechungen, verschiedenartige äußere Rahmenbedingungen (Streiks, Verkehrssystem), gegensätzliches Kommunikationsverhalten sowie der beidseitige Überlegenheitskomplex genannt. Gehen wir von kulturell ähnlichen Grundannahmen in diesen beiden Länder aus (vgl. z.B. die Cluster-Studien von Hofstede, 1980), so mögen die artifiziellen kulturellen Differerenzen für ein interkulturelles Management zwar kompliziert erscheinen (wie es das Verhältnis von Franzosen und Deutschen schon immer war), es aber keiner neuen Handlungstheorien bedarf, sondern diese durch Neugestaltung bzw. Abstimmung von Zielen, Aufmerksamkeitsregeln und Problemlösungs verfahren (March/OIsen, 1976) zu handhaben sind.

Einige gegenwärtig noch nicht abgeschlossene Untersuchungen zum Vergleich von Managementstilen in deutschen und britischen Unternehmen Iassen ähnliche Befunde erwarten. Es deuten sich hier aber auch Ergebnisse an, die darauf hinweisen, daß der zuvor angesprochene "Korridor" an der Kulturoberfläche enger sein kann als auf den tieferen Ebenen ${ }^{24}$. So verfügen die englischen Manager über ähnliche formale Instrumente wie die

Die JPB - La Synergie Franco-Allemande befragte 1989 schriftlich und per Telefoninterview 200 Mutter- und Tochtergesellschaften in Frankreich und Deutschland, bezüglich Problemen alltäglich geschäftlichen Umgangs mit der jeweilig anderen Kultur (vgl. Breuer, 1990: 22ff).

Ein ähnliches Problem wird auch in der Studie Hall/Hall (1991: 135) herausgearbeitet. Die Ergebnisse beruhen auf Interviews mit den Führungen deutscher, französicher und amerikanischer Unternehmen und bestätigen i.w.S. mittels einer pointierten und sehr differenzierten Betrach tungsweise die Cluster-Studien von Hofstede.

Dies ist auf den ersten Blick zumindest deshalb überraschend, weil üblicherweise davon ausgegangen wird, daß stark differente Normen und Werte ein noch höheres Maß an unterschiedlichen Artefakten erzeugen Dies betonen z.B. Adler/Graham:"The greater the cultural differences, the more likely barriers to communication and misunderstandings become." (1989: 517) Wie auch an Beispielen aus dem japanischen Management noch zu zeigen sein wird, lassen sich jedoch entgegengesetzte Fälle ebenso beobachten. In diesem Falle verstellen die Ähnlichkeiten auf der Ebene der Kultursymbole den Blick für entscheidende Differenzen auf den Ebenen der Normen und Werte (vgl. hierzu auch unsere Ausführungen zur Kulturgebundenheit von Management im 2. Abschnitt) 
deutschen (z.B. Stellenbeschreibungen), weisen ihnen jedoch eine andere (höhere oder geringere) Bedeutung zu, als ihre deutschen Kollegen. ${ }^{25}$ Ähnlichkeiten auf der artifiziellen Ebene sind hier mit Un terschieden auf der Normenebene verbunden.

Mit dem zweiten Beispiel soll das Beharrungsvermögen "basaler Annahmen" illustriert und der Einfluß der Ethno- auf die Wirtschaftskulturen verdeutlicht werden.

Die gegenwärtige Entwicklung in Osteuropa liefert hier geradezu ein Paradebeispiel, zeigt sie doch (etwa in J ugoslawien und der ehemaligen Sowjetunion) eindrücklich die Stärke von National- und Ethnokulturen auf. Diese sind offenbar auch durch eine mehr als ein halbes J ahrhundert währende Zwangssozialisation auf übergeordnete Einheitskulturen (besser: Ideologien) nicht zu brechen. Wie diese basalen Umgebungskulturen auch in den Wirtschaftsbereich hineinwirken und somit auf Unternehmenskulturen Einfluß nehmen, zeigt Dülfer (1991) anschaulich auf. So beeinflussen etwa die "belief systems" fundamentalistischer Religionen (Islam) in starkem Maße den Arbeitsrhythmus, der ausschließlich durch Tag-Nacht- und Jahreszyklen bestimmt wird. Damit verschwinden zeitliche Opportunitätskosten, die in westlichen Kulturen hingegen eine große Rolle spielen (Dülfer, 1991: 266 ff.).

Mit dem dritten Beispiel wenden wir uns dem Problembereich zu, der die Diskussion um kulturbezogenes Management überhaupt erst in Gang gesetzt hat - dem Vergleich östlicher und westlicher Managementstile.

$\mathrm{N}$ ach der in der Frühphase der Management-Kulturforschung nahezu einhellig vertretenen Meinung, basieren "Kunst" und Überlegenheit des japanischen Managements bekanntlich auf einer starken Unternehmenskultur, d.h. auf gemeinsam geteilten Grundannahmen mit ausgeprägtem Verpflichtungscharakter für jedes einzelne Organisationsmitglied. Aus westlicher Perspektive wurde dies vorwiegend als "hohe Identifikation" und "weiches" Management interpretiert.

Diese weichen Faktoren können jedoch wohl nur aufgrund unserer kulturellen Deutungsmuster als "weich" bezeichnet werden. Im Kontext der japanischen Kultur sind sie vielmehr als die eigentlich harten Faktoren anzusehen. Wir interpretieren sie individualistisch und verbinden damit die Eröffnung individueller Handlungs- und Wahlmöglichkeiten sowie eine Unterstützung des Wertes der Selbstverwirklichung. Im Rahmen der japanischen Kultur sind sie Medium zur Verpflichtung Einzelner auf die übergeordneten kollektiven Werte und Interessen, wobei die Bindung/ldentifikation durch soziale Sanktionen sichergestellt wird, die unseren Vorstellungen kaum entsprechen. Für 
unser Weltbild bedeuten sie deshalb eher "Differenzierung", für das japanische eher "Integration".

Die Dominanz kollektiver Werte kann als ein "Erfolgspotential" angesehen werden, das japanischen Unternehmenskulturen von ihrer Umgebungskultur "in die Wiege gelegt" wurde. Wie lange dieses Erfolgspotential wirkt, hängt auch von der Geschwindigkeit des Wertewandels ab. Dieser vollzieht sich im Westen deutlich schneller als in J apan. Dennoch: J apan ist ein Beispiel für die Wirksamkeit der Wirtschaft als Katalysator des kulturellen Wandels. Denn mögen die Japaner es auch geschickt verstehen, ihre Warenmärkte zu schützen, für Kulturimport gibt es kaum eine Gegenwehr. Einen mit dem Güterexport gleichzeitig stattfindenden Werteimport kann man kaum verhindern. So wird mittlerweile nicht nur deutlich, in welchem dramatischen Umfang kollektive Kulturen Streß erzeugen ${ }^{26}$, sondern auch, daß für die junge japanische Generation individualisierte westliche Wertstrukturen ein großes Attraktivitätspotential besitzen. Der Preis des wirtschaftlichen Erfolges besteht langfristig gesehen vielleicht gerade darin, die Wertstrukturen zu verlieren, die ein wesentlicher Garant dieses Erfolges waren.

Einige Untersuchungen zur Personalpolitik in deutschen Tochtergesellschaften japanischer Unternehmungen lassen vermuten, daß es japanischen Unternehmen im Gegenzug offensichtlich nur wenig gelingt, über ihre Managementkultur auch Elemente ihrer Nationalkultur zu exportieren. J apanisches Management im ursprünglichen Sinn scheint es, in diesen deutschen Tochtergesellschaften kaum zu geben. Gleichwohl sind Schwierigkeiten der Zusammenarbeit mit japanischen Kollegen und/oder Vorgesetzten im wesentlichen auf größere Unterschiede auf der Normen- und Wertebene zurückzuführen (Heidrick \& Struggles, 1990; Sieboldt, 1992).

Die genannten Beispiele weisen nicht nur auf die verschiedenen Konfliktpotentiale entlang des Kulturkorridors hin, vielmehr machen sie auch deutlich, wie kontraproduktiv der Versuch sein kann, erfolgreiche Handlungstheorien und Lernkonzepte fremder Kulturen zu kopieren, ohne sie zu hinterfragen.

Zur Veranschaulichung dieses Problems bietet sich aus aktuellem Anlaß das Konzept der "lean production" an - in der Praxis gegenwärtig wohl das Managementthema schlechthin.

Die managementphilosophische Klammer hinter den Bemühungen um das Phänomen der "lean-production" wird unter dem Begriff des "Kaizen" zusammengefaßt. Dies bedeutet ständige Verbesserung durch und zum Wohle aller. Für den "Rest der Welt" ist dieses Konzept durch die Studie von Womack/J ones/Roos (1991) stark ins Bewußtsein gerückt

26 Entsprechende Informationen haben sich in letzter Zeit v.a. durch die Diskussion um das "Iean management" verbreitet. 
worden $^{27}$. Sie zeigt, mit welchen Produktionsvorteilen japanische Automobilkonzerne gegenüber ihren westlichen Konkurrenten ausgestattet sind. Was liegt näher als der Versuch, diese offensichtlich erfolgreiche Kaizen-Strategie zu kopieren. Betrachtet man die Instrumente näher, mit denen "Iean production" realisiert wird, kann man zunächst zu dem oberflächlichen Resultat kommen: es handelt sich hierbei im wesentlichen um "kulturfreie" Methoden. Die Unterschiede liegen jedoch in der die "lean production" tragenden Managementphilosophie. An dieser Stelle zeigt sich wie geeignet dieses Beispiel ist für die von uns zuvor behauptete Kulturüberformung des Managements. Das Besondere an der "Iean production" sind nicht die Instrumente, sondern vielmehr die Art und Weise, wie diese integriert und durchgesetzt werden, sowie die Intensität, mit der sich ganz offenkundig Manager und Arbeiter auf "Kaizen" verpflichten Iassen. Das tragende Wertgerüst, und damit der Kern der Managementphilosophie, ist eine stabile und unwidersprochen an Kollektivinteressen ausge richtete soziale Hierarchie.

Aus Sicht eines europäischen Wertesystems zeigt sich "Iean production" als eine Art "partizipativen Verbesserungslernens", wobei sich die Partizipation auf die Verfahrens-, aber nicht auf die Ziele und Werteebene bezieht. Versucht man nun, diese "lean production"Instrumente z.B. in Form des mittlerweile auch bei uns populär gewordenen Schlagwortes "Iean management" zu übertragen, wird man vermutlich feststellen, daß die Gesellschaftskultur gegen den Import der japanischen "Iean management"-Philosophie weitgehend resistent ist, da die darin enthaltenen basalen Annahmen (Werte) inkompatibel sein dürften. Der Versuch, die "lean"-Philosophie unangepaßt auf unser europäisches Wertesystem zu übertragen, wie dies offensichtlich einige Unternehmen der Automobil- und Automobilzulieferbranche tun, birgt ein hohes Risiko des Scheiterns. Es sind folglich Transformationsleistungen durch interkulturelle Lernsysteme (deutero learning) ${ }^{28}$ notwendig, um derartige Managementsysteme überhaupt erfolgreich übertragen zu können. ${ }^{29}$ Finden wir diesen kulturellen Transformationsschlüssel nicht, werden sich bei der Übertragung des "Iean management" vermutlich westliche Alternativnormen zur östlichen Gruppenverpflichtungskultur "wie von selbst" einstellen. Da eine solche westliche Alternative zur kollektiven Selbstverpflichtung die formale Fremdverpflichtung ist, dürfte

In J apan hat diese Studie im übrigen weit weniger Aufsehen erregt. Vielmehr sind dort auch die Grenzen dieses Konzepts mittlereweile deutlich hervorgetreten (Streß, schlechte Arbeitsbedingungen, geringe intrinsische Motivation der Mitarbeiter). Vgl. hierzu etwa die Ergebnisse des Kongresses, den die IG Metall im J uni dieses J ahres durchführte (vgl. Nomuri, M. 1992, Toyo tismus am Ende?) innerhalb seines kulturellen Kontextes und die eigentliche Transformation in eine andere Kultur. Sie müssen folglich über zwei "Schlüssel" verfügen.

Wie allgemein bekannt, gibt es natürlich auch Beispiele, wo diese Übertragung erfolgreich durchgeführt wurde. Allerdings geben die diesbezüglichen Studien keine Auskunft darüber, inwieweit tatsächlich ein Transfer der typisch japanischen Managementphilosophie stattgefunden hat. Vielmehr deutet einiges darauf hin, daß die hier angeführten Beispiele in besonders "günstigen" Situationen stattfanden. Die meisten Beispiele beziehen sich auf Firmenneugründungen und damit auf die Neuschaffung einer Firmenkultur und nicht auf eine grundsätzliche Veränderung. Außerdem läßt sich vermuten, daß bestimmte Rahmenfaktoren, z.B. hohe Arbeitslosigkeit und die Infrastrukturpolitik der jeweils betroffenen Region unterstützend wirkten. Zudem sind die Erfahrungszeiträume noch außerordentlich kurz. 
sich hier die Bürokratie als Steuerungsäquivalent anbieten. "Lean Management" würde dann also etwas erzeugen, das durch seinen Einsatz eigentlich abgebaut werden sollte. Daß sich in diesem Fall des "interkulturellen Nicht-Lernens" eine außerordentlich kontraproduktive Emergenz ergibt, muß nicht weiter aus gef ührt werden. 


\title{
4. Ansatzpunkte f ür ein Management des interkulturellen Lernens
}

Nachdem bisher die analytischen und die konzeptionellen Aspekte des interkulturellen Lernens im Vordergrund standen, wird mit den nachfolgenden "Ansatzpunkten für das Management" die Gestaltungsperspektive in den Mittel punkt gerückt.

\author{
Aktionsschwerpunkte und "Selbstverständnis" eines Managements des \\ interkulturellen Lernens \\ Aus dem zuvor entwickelten "Orientierungsmodell" Iassen sich zwei grundsätzliche \\ Aktionsschwerpunkte für ein Management des interkul \\ turellen Lernens ablei ten: \\ - Ausrichtung der Aktivitäten auf Divergenzen und Gemeinsamkeiten entlang des \\ "Kultur-Korridors" \\ - Entwicklung von Strategien des "deutero learning"
}

Mit diesen Schwerpunktsetzungen sind bereits Vorentscheidungen für das Sel bstverständnis und die spezifische inhaltliche "Qualität" eines sol chen Managements getroffen. $\quad 30$

Die Orientierung an interpretativen Kulturkonzepten impliziert, daß ein solches Management "kulturbewußt" agiert, statt sich an mechanistischen Machbarkeitsvorstellungen auszurichten.

Die Ausrichtung auf Konzepte des "organisationalen" Lernens ermöglicht, Management als ein "sich selbst organisierendes Phänomen" anzusehen - als eine "Eigenschaft des Systems" und nicht als einen klar abgrenzbaren und personell eindeutig zuordnungsfähigen Teil der Organi sation.

Eine Berücksichtigung von Divergenzen (und Gemeinsamkeiten) auf den verschiedenen Kulturebenen erfordert die Fähigkeit, sich "kulturüber-greifend" mit unterschiedlichsten Wertkonstellationen auseinandersetzen zu können. Eine "ethnozentrische" $\mathrm{Handlungstheorie} \mathrm{wird} \mathrm{den} \mathrm{Bedingungen} \mathrm{des} \mathrm{an} \mathrm{"Kulturdifferenzen"} \mathrm{ausgerichteten}$ organisationalen Lernens kaum gerecht. "Polyzentrisch" oder gar "geozentrisch" ausgerichtete Handlungstheorien führen jedoch zu einem Management, dessen Hauptaufgabe darin besteht, zwischen uniformierenden und differenzierenden Tendenzen zu vermitteln und diese immer wieder neu auszu-balancieren. ${ }^{31}$ "In fact the more conforming the society is,

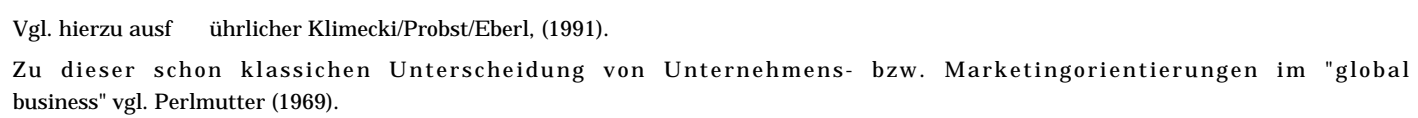


the more it creates differencies. ... Every tendency creates a counter-tendency. ... There is always a differentiation as well as a uniformization ..." (Kotler, 1990: 87).

Globales Management ist deshalb, so widersprüchlich es auch auf den ersten Blick erscheinen mag, "differentielles" Management (vgl. Evans/Doz, 1989: 219ff).

Schneider (1988) zeigt drei Grundprobleme eines solchen differentiellen Manag

ments auf:

- Diffenzierung vs. Integration: Überlagerungen zwischen Unternehmenskulturen, die nach Vereinheitlichung streben, und länderspezifi schen Kulturunters chieden.

- Zentralismus vs. Autonomie: Widersprüche, die zwischen den globalen Visionen der Unternehmensphilosophie - als "zentralistischer" Steuerungsansatz - und den Autonomienotwendigkeiten und (-bedürfnissen) der Ländereinheiten bestehen können.

- Nationale vs. Unternehmensorientierung: Widersprüche zwischen kosmopolitischen und Iänderspezifischen Orientierungen (z.B. in der Personalarbeit, insbesondere bei grenzüberschreitender Führungs $\quad k r$ äfte entwicklung).

\section{Ein "magisches Dreieck" als Ordnungsrahmen für das Management des interkulturellen Lernens}

Neben die zuvor (zunächst noch abstrakt) aufgezeigten Aktionsschwerpunkte und das "Selbstverständnis" eines Managements des interkulturellen Lernens wollen wir - als drittes Element - ein Ordnungsschema stellen. Wir verwenden dafür eine Modifikation des in der modernen Managementlehre gegenwärtig recht beliebten "magischen Dreiecks". Es stellt ein didaktisches Hilfsmittel dar, um die Aktionsfelder des Managements und deren Wechselwirkungen zu verdeutlichen.

In weiten Teilen der Globalisierungsliteratur findet dieses Dreieck (zumindest implizit) ebenfalls Verwendung. Die aus der Globalisierung resultierenden Probleme werden dabei in ein Spannungsfeld gestellt, das durch die "Eckpunkte" Strategie, Struktur und Kultur abgegrenzt wird. Das "Königsinstrument" und damit - bildlich gesehen - als der Mittelpunkt des Dreiecks, wird üblicherweise das Human Resources Management (HRM) angesehen. Dies ist mit Blick auf die Probleme des qualitativen Wachstums bzw. der "inneren Größe" eine naheliegende Schwer punktsetzung.

Die Gestaltungsempfehlungen zielen in der Regel darauf ab, über Entwicklung von Humanressourcen die "interne Integration" (Schein) und, damit verbunden, die I mplementationsprobleme $\quad{ }^{32}$ globaler Wettbe werbsstra tegien "in den Griff" zu bekommen. ${ }^{33}$

Aus der Perspektive des interkulturellen Managements empfiehlt es sich, das oben beschriebene Dreieck anders zu arrangieren (Abb.5).

Die Lösung des Implementationsproblems ist, überspitzt formuliert, fast wichtiger als die Wettbewerbsstrategie selbst (vgl. hierzu den Beitrag von Meffert in diesem Band).

33 Vgl. Adler/Ghadar, 1990; Pieper, 1990; Pucik, 1988; Schulte, 1988; Laurent, 1986; Evans, 1986. 
Für diese Neuordnung sind fol gende Gründe ausschlaggebend:

- aus dem hier vertretenen Management-L eitbild (Selbstverständnis) resultiert, daß Kultur nicht "gleichrangig" neben die anderen Aktionsfelder gestellt weden kann, sondern den gemeinsamen Bezugspunkt und die Interpretationsgrundlage aller Managementaktivitäten darstellen muß. Es gilt, sämtliche Managementaktivitäten vor dem Hintergrund möglicher Kulturdivergenzen zu evaluieren und ihre Implementation "differentiell" auf die Erhaltung und/oder Überbrückung kultureller Unterschiede bzw. auf ein damit verbundenes Konfliktmanagement aus zu rich ten.

- Kultur ist kein Aktionsfeld sondern, wie es der interpretative Ansatz nahelegt, eine "Metapher" für Wahrnehmungs- und Bewertungsvorgänge (vgl. Klimecki/Probst 1991: 43). Sie auf der gleichen Ebene wie Struktur und Strategie anzusiedeln, vermittelt zu stark den Eindruck, sie auch auf die gleiche Weise gestalten zu können.

- Vorliegende Empfehlungen zum HRM im Rahmen des globalen Managements Iassen sich (vielleicht etwas überpointiert) auch als Hilfsstrategien für die Realisierung von Unternehmens- oder (marktbezogenen) Wettbewerbsstrategien interpretieren (vgl. Meffert, 1989). Die zuvor angesprochene Königsfunktion des HRM wäre dann die eines "ersten Dieners": Dies scheint uns mit Blick auf die Entwicklung einer "Global Work Force" (J ohnston, 1991) nicht erfolgversprechend. Vielmehr lassen einige Szenarien vermuten, daß die Arbeitsmärkte weltweit ein eigenständiges Globalisierungsproblem erzeugen, das jenes der Waren- und Dienstleistungsmärkte hinsichtlich Komplexität und "Management-I mpact" noch über treffen wird. 


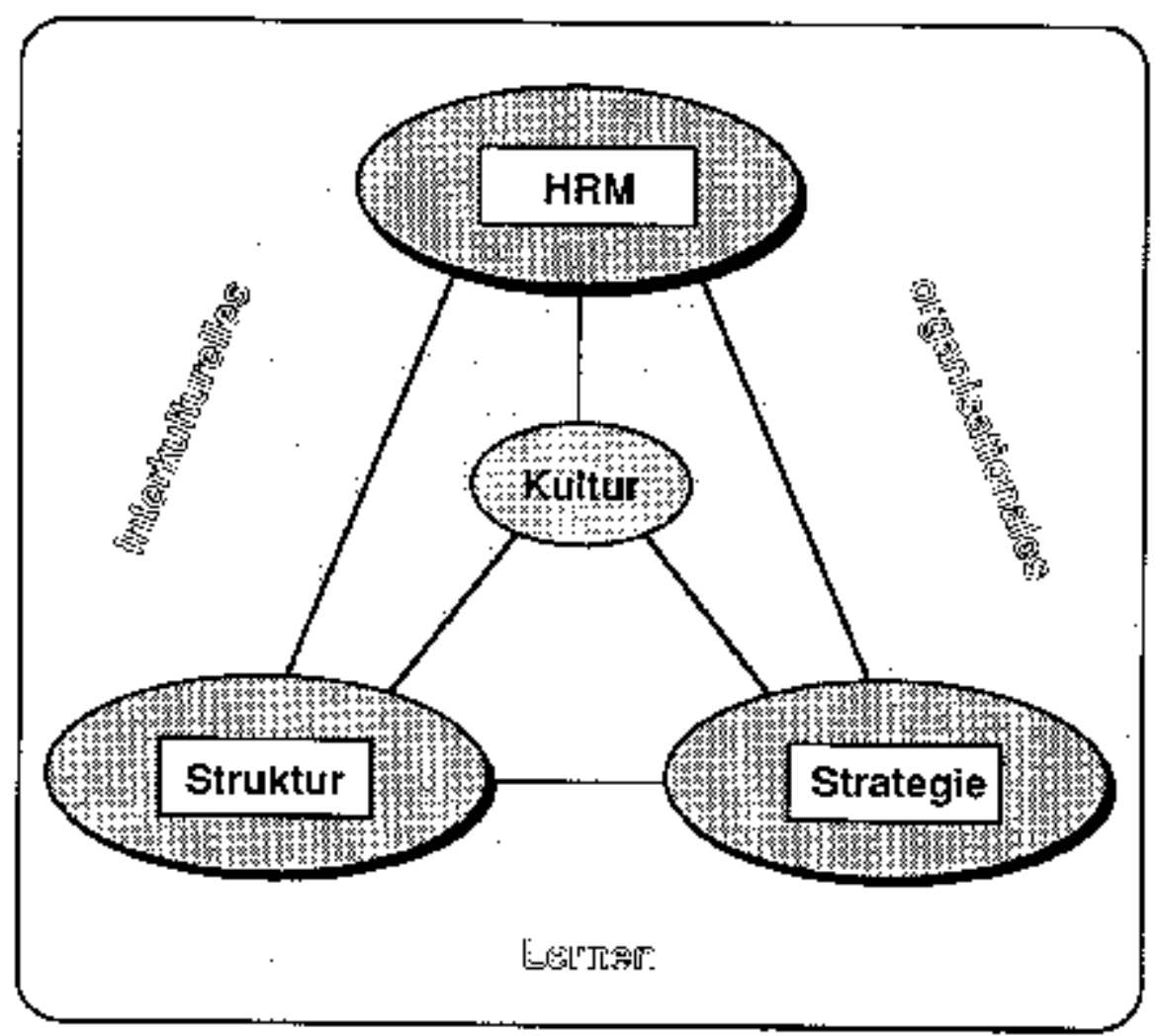

\section{Abb.5 "Magisches-Dreieck" als Ordinugsrahmen interkulturell organisationaten Lernens}

Abb.5 "Magisches-Dreieck" als Ordnungsrahmen interkulturell organisationalen Lernens

- Wir sehen HRM deshalb als ein eigenständiges Aktionsfeld an, das den übrigen Feldern nicht hierarchisch nachgeordnet, sondern mit ihnen netzwerkartig verbunden ist und dessen "Königsfunktion" aus folgenden Effekten resultiert:

- Individuelle Entwicklungsmotivation und Kommunikationsfähigkeit sind die wichtigsten "Medien" des organisationalen Lernens.

- Persönliche Werthaltungen, Interessen und Ansprüche stellen sich als die bedeutsamsten Knotenpunkte im "unternehmens-kulturellen N etz" dar.

- (Internationale) Arbeitsmärkte werden aufgrund ihrer brisanten Entwicklung verstärkt zu einem eigenständigen und hochrangigen Managementproblem.

Auf einige dieser Entwicklungen sei in Anlehnung an J ohnston (1991) kurz hingewiesen: Massiver Eintritt von Frauen in den Arbeitsmarkt; steigendes Durschnittsalter hochqualifizierter Arbeitnehmer mit ebenfalls steigendem Anspruchsniveau; Angleichung des Bildungsstandes (zumindest junger Arbeitnehmer) in Entwicklungs- und Industrienationen; aus den Entwicklungsländern drängt eine wachsende Zahl hochqualifizierter Arbeitssuchender auf den Welt-Arbeitsmarkt; aufgrund des Nord-Süd-Lohngefälles kommt es zu Abwanderungen größeren Ausmaßes, in deren Folge verlieren die Entwicklungsländer einen großen Teil des dort so dringend benötigten Humankapitals. Als weitere Folge entsteht ein starker Druck (nicht nur) auf die "middle income"-Nationen, verbunden mit einer massiven Emigrantenproblematik. 


\section{"Intercultural Pathfinding" als Strategieempfehlung}

Das "Erfolgspotential", an dem sich interkulturelle Lernstrategien langfristig ausrichten können, ist die Überbrückung von Kulturdivergenzen und das Management von Kulturkonflikten.

Wenn davon auszugehen ist, daß Kulturunterschiede kommunikative Barrieren verstärken und für Mißverständnisse ursächlich sind, werden Sensibilität für und Analysefähigkeit von Kulturdifferenzen sowie der Aufbau interkultureller kommunikativer Kompetenz zu einem Schwerpunkt thema dieser Lernstrategien.

Da diese Gratwanderung zwischen den Kulturen in Überschneidungssituationen zwischen eigen- und fremdkulturell geprägten Denk- und Deutungsmustern, Emotionen und Verhaltensweisen stattfindet, ist die Kenntnis der jeweils zugrunde gelegten Orientierungsund Symbolsysteme für eine Überbrückung von Divergenzen unabdingbar (Thomas, 1990: 149). Diese Muster sind in globalen Handlungsfeldern jedoch nicht exakt vorhersehbar, deshalb müssen Lernformen eingesetzt werden, mit deren Hilfe "... generalizable skills by which individuals 'learn how to learn' in any intercultural setting..." (Lobel, 1990: 44) nicht nur "individuell antrainiert", sondern in die "kognitiven Landkarten" der Organisation Eingang finden und damit auch organisationsweit kommuni kationsf ähig werden.

Die Erfolgspositionen interkultureller Lernstrategien Iassen sich folglich inhaltlich nur schwer bestimmen. Sie bestehen darin, Entwicklungspotentiale freizusetzen und Prozesse der Selbstorganisation anzuregen. Der "Motor", der dynamische Aspekt solcher Entwicklungsstrategien, ist das (organisationale) Lernen "zweiter Ordnung". Aus Iernstrategischer Sicht gilt es daher, die Entwicklungskräfte auf die Förderung von "deuterolearning"-Kompetenzen zu konzentrieren.

Das Objekt dieser Strategien des "Lernens, wie man lernt" kann als "intercultural pathfinding" bezeichnet werden, da es im wesentlichen darum geht, "Pfadfinderstrategien" zu entwickeln, wie man sich im "Dschungel interkultureller Divergenzen" zurechtfinden kann. 


\section{"Reflective Practitioning" $\quad{ }^{35}$ als Strukturempfehlung}

Lernstrategien der "zweiten Ordnung" kosten Zeit und erzeugen Opportunitäts- sowie Transaktionskosten. Sie setzen auch ein nicht zu unterschätzendes Maß an "organizational slack" (Simon) voraus. Dies ist im Zeitalter schlanker Strukturen jedoch eine Forderung, die auf Widerspruch stoßen muß, da alle Energien, die nicht unmittelbar auf die Bewältigung der primären Organisationsaufgaben gerichtet sind, als "vergeudet" angesehen werden müssen. Dennoch sind solche "Vergeudungen" notwendig; denn nur sie ermöglichen Deutero-Lernstrategien. Da diese jedoch nicht instrumentell sind, d.h. nicht unmittelbar auf die Lösung von Organisationsproblemen ausgerichtet werden können, bedürfen sie einer besonderen Begründung. Diese ist darin zu finden, daß die primären Organsationsaufgaben in "turbulenten Zeiten" auf Dauer nur dann zu erfüllen sind, wenn die Chance besteht, die bisherigen $\mathrm{H}$ andlungstheorien hinsichtlich ihrer zukünftigen Brauchbarkeit hinterfragen zu kön nen. ${ }^{36}$

Der Erfüllung primärer Organisationsaufgaben wird damit zwar kurzfristig Energie entzogen, mit der jedoch langfristig ihre Erfüllung gesichert werden soll. Dieses "venture capital" des organisationalen Lernens kann deshalb nicht anhand von "Lageindikatoren" (z.B. Produktivitätskennziffern) evaluiert werden. Das entscheidende Beurteilungskriterium ist vielmehr der nur langfristig und häufig erst ex-post erkennbare Zugewinn an Handlungskompetenz oder "innerer Größe".

Die institutionellen Voraussetzungen für eine sinnvolle "Verzinsung" dieses Risikokapitals zu schaffen, ist eine der wesentlichen Aufgaben von Strukturkonzepten für organisationales Lernen. Dabei gilt es, Freiräume anzubieten, in denen ein "reflective practitioning" stattfinden kann. Moderne (interaktiv ausgerichtete) Personalentwicklungskonzepte ${ }^{37}$ zei- $^{-}$ gen auf, daß dies dauerhaft nur durch eine Verbindung von Arbeits- und Lernwelt erreicht werden kann. Dazu sind Teamstrukturen erforderlich, die Prozessen der Selbstreflexion nicht nur Raum gewähren, sondern diese zu einem arbeitsorganisatorischen Prinzip erklären. 38

\section{"The missing link": Interkulturelles Human Resources Management}

Wenn wir dem HRM im Einklang mit der vorherrschenden Meinung einerseits eine "Königsfunktion" zusprechen und es andererseits als ein "missing link" bezeichnen, so resultiert dieser Widerspruch aus einem U.E. noch nicht überwundenen Dilemma, das vermutlich eine Übergangserscheinung zwischen verschiedenen Paradigmen der Personalar-

Der Begriff geht zur ück auf ein von D. Schön vorgestelltes Konzept der "reflexiven Praxis" (vgl. Schön,1983).

Vgl. hierzu ausf ührlicher; Klimecki/Probst/Eberl (1991) und Staehle (1991).

Vgl. exempl. die Publikationen von Sattelberger (1989, 1991, 1992).

Auf eine Illustration solcher Organisationsformen wird hier bewußt verzichtet. Teilautonome Arbeitsgruppen sowie Fertigungsinseln eignen sich dazu ebenso wie projektorientierte Organisationsformen. Auch hier kommt es weniger auf das "was", als auf das "wie" an. 
beit darstellt. ${ }^{39}$ Aus klassischer Sicht ist das Personal eine an Organisationsziele und strukturen anzupassende "Variable". Gerade die Globalisierungsproblematik zeigt jedoch, daß faktisch über internationale Strategien erst dann sinnvoll zu diskutieren ist, wenn deren "personale Basis" sichergestellt werden kann. Die abhängige Variable wird immer mehr zu einer Gestaltungsvoraussetzung. Die Implementation von Strategien und Strukturen des interkulturellen Lernens ist ganz entscheidend davon abhängig, inwieweit es gelingt, sie durch kongeniale HRM-Konzepte zu "vitalisieren". Diese Notwendigkeit ist im Bereich globaler Wettbewerbsstrategien auch schon seit Iängerer Zeit erkannt worden. ${ }^{40}$ Aus naheliegenden Gründen wird das HRM hier jedoch vorwiegend noch aus der Anpassungsperspektive betrachtet (die behauptete Vormachtstellung wird faktisch zu einer "Hab'-Acht"-Stellung). Die Literatur zum internationalen Personalmanagement ${ }^{41}$ beharrt hingegen weitgehend auf einer individualistischen Perspektive und konzentriert sich auf Analysen und Gestaltungsvorschläge, in denen der Kontext des HRM (z.B. strukturelle Barrieren oder die angesprochenen "defensive routines") nur eine untergeordnete Rolle spielt bzw. als "unzureichend" beklagt wird (vgl. z.B. Lichtenberger, 1992: 197f).

Die Lösungsansätze eines interkulturellen HRM favorisieren meist zwei Aktionsbereiche. Zum einen geht es um die kulturübergreifende Qualifikation von Managern (möglichst mit dem Ziel des "just one world"-Managers) und zum anderen um die tragende Funktion des HRM für die Ausbildung einer adäquaten Unternehmenskultur. Dabei wird, wie bereits aufgezeigt, grundsätzlich empfohlen, die HRM-Strategien der jeweils verfolgten Gesamtstrategie anzupassen.

Bei der Realisierung dieser HRM-Strategien stehen vor allem klassische Instrumente und Funktionsbereiche wie Auswahl, Einsatz, Entwicklung usw. im Vordergrund (vgl. z.B. Pucik, 1988). ${ }^{42} \mathrm{Hier}$ wird in der Literatur weitgehend unterstellt, daß diese Instrumente kulturwirksam sind (vgl. u.a. Earley, 1987: 687; Pucik, 1988: 81; Thomas, 1990: 150). Und im weiteren wird davon ausgegangen, daß es notwendig ist, eine übergeordnete integrative Unternehmenskultur zu schaffen, die auf globale Erfordernisse ausgerichtet ist und gleichsam regionale kulturelle Eigenarten berücksichtigt (vgl. Ronen, 1986: 231f, 267). Dies ist insgesamt plausibel, läßt jedoch weitgehend die Frage außer acht, welche Kulturen für die Bewältigung globaler Managementprobleme benötigt werden, in welcher Weise sich durch die genannten Personalstrukturen diese Arten von Unternehmenskultur herstellen Iassen, welche (national geprägten) Werte das "Personal" in die Organisation einbringt und welche Kulturkonflikte damit wiederum verbunden sind. (Nicht nur) Bezogen auf das Problem des

39 Vgl. ausf ührlicher Klimecki/Probst (1992).

40 Zum Überblick vgl. etwa Meffert (1989).

41 Vgl. hierzu aus dem Reader: Pieper (Hrsg.): Human Resource Management. An international Comparison (1990) u.a. die Beiträge von Staehle, Dülfer und Adler/Gadhar, sowie aus dem Reader: Evans/Doz (Hrsg.) HRM in International Firms (1989) u.a. die Beiträge von Evans/Lank/Farquhar und Evans/Lorange. alle vom Training über J ob Rotation bis hin zum Mentoring "interkulturalisiert" worden, jedoch ohne diese in einen übergeordneten (personal politischen) Rahmen einzufügen. 
interkulturellen Lernens illustriert die noch wenig ausgebaute Vernetzung von Personalund Unternehmensentwicklung deshalb ein wesentliches "missing link" des globalen Managements. 


\section{Literaturverzeichnis}

Adler, N. (1983) A Typology of Management Studies Involving Culture. In: J ournal of International Business Studies, Fall, S. 29-43.

Adler, N./Ghadar, F. (1990) Strategic Human Resource Management: A Global Perspective. In: Pieper, R. (Hrsg.) Human Resource Management. An International Comparison. Berlin, New York.

Adler, N./Graham, J. (1989) Cross-Cultural Interaction: The International Comparison Fallacy? In: J ournal of International Business Studies, Fall, S. 515-537.

Argyris, C. (1990) Overcoming Organizational Defenses. Boston u.a.

Argyris, C./Schön, D. (1978) Organizational Learning: A Theory of Action Perspective. Boston.

Bartlett, C.A./Ghoshal, S. (1989) Managing Across Borders. Boston.

Bateson, G. (1981) Ökologie des Geistes. 2. Aufl. Frankfurt.

Black, J .S./Mendenhall, M. (1990) Cross-Cultural Training Effectiveness: A Review and a Theoretical Framework for Future Research. In: Academy of Management Review, Vol. 15, No. 1, S. 113-135.

Breuer, J.P. (1990) Syntonie und Interface. Konfliktlösungs-Service für deutschfranzösische Kooperationen. (J PB-Studie) In: Marktforschung \& Management 1/90, S. 2226.

Brislin, R.W. (1989) Intercultural Communication Training. In: Asante, M.K./Gudykunst, W.B. Handbook of I nternational and I ntercultural Communication. London. S. 441-457.

Brislin, R.W./Bochner, S./Lonner, W.J . (1975) Cross-Cultural Perspectives on Learning. New York.

Brislin, R.W./Cushner, K./Cherrie, C./Yong, M. (1986) International Interactions - A Practival Guide, Volume 9, Cross-Cultural Research and Methodology Issues. Beverly Hills.

Davis, S.M. (1989) Future Perfect. In: Evans, P./Doz, Y./Laurent, A. Human Resource Management in International Firms - Change, Globalization, Innovation. Houndmills u.a. S. 18-29.

Dülfer, E. (1990) Human Resource Management in Multinational and International Operating Companies. Pieper, R. (Hrsg.) Human Resource Management. An International Comparison. Berlin, New York. S. 261-285.

Dülfer, E. (1991) Internationales Management in unterschiedlichen Kulturbereichen. München.

Duncan, R./Weiss, A. (1979) Organizational Learning: Implications for Organizational Design. In: Research in Organizational Behavior, Vol. 1, S. 75-123.

Early, P.C. (1987) Intercultural Training for Managers: A Comparison of Documetary and Interpersonal Methods. Academy of Management J ournal Vol.30, Nr.4, S. 685-698. 
Evans, P./Doz, Y./Laurent, A. (1990) Human Resource Management in International Firms - Change, Globalization, Innovation. Houndmills u.a.

Fiol, C.M./Lyles, M.A. (1985) Organizational Learning. In: Academy of Management Review Vol.10, Nr.4, S. 803-813.

Habermas, J . (1982) Theorie des kommunikativen Handelns (I). Frankfurt.

Hall, E.T./Hall, M.R. (1990) Understanding Cultural Differences. Yarmouth.

Harris, P.R./Moran, R.T. (1982/91) Managing Cultural Differences. 1d and 3d Edition. Houston.

Hedberg, B. (1981) How Organizations Learn and Unlearn. In: Nystrom, P.C./Starbuck, W.H. (Hrsg.) Handbook of Organizational Design. London. S. 8-27.

Heidrick \& Struggles (1990) Japanische Unternehmen in der Bundesrepublik Deutschland und ihre Personalsituation. Gesellschaft für Wirtschaftsförderung Nordrhein-Westfalen (Hrsg.) Düssel dorf.

Hofstede, G. (1980) Cultures' Consequences. International Differences in Work-Related Values. Beverly Hills.

Hofstede, G. (1983) The Cultural Relativy of Organizational Practices and Theories. In: J ournal of International Business Studies, Fall, S. 75-89.

Imai, M. (1992) Kaizen. Der Schlüssel zum Erfolg der J apaner im Wett bewerb. M ünchen.

J ohnston, W.B. (1991) Global Work Force 2000: The New World Labor Market. Harvard Business Review, March-April, S. 115-127.

Kasper, H. (1987) Organisationskultur. Wien.

KiechI, R. (1990) Ethnokultur und Unternehmenskultur. In: Lattmann (Hrsg.) Die Unternehmenskultur. Heidelberg. S. 107-130.

Klimecki, R.G./Probst, G.J.B. (1992) Integrierte Personal- und Organisationsentwicklung. In: Lattmann, C./Probst, G./Tapérnoux, F. (Hrsg.) Die Förderung der Leistungsbereitschaft des Mitarbeiters als Aufgabe der Unternehmensführung. Heidel berg.

Klimecki, R.G./Probst, G.J.B. (1990) Entstehung und Entwicklung der Unternehmenskultur. In: Lattmann (Hrsg.) Die Unternehmenskultur. Heidel berg. S. 4167.

Klimecki, R.G./Probst, G.J.B./Eberl, P. (1991) Systementwicklung als Managementproblem. In: Staehle, W.H./Sydow, J. (Hrsg.) Managementforschung 1, Berlin, New York. S. 103-162.

Kotler, P. (1990) Globalization - Realities and Strategies. In: Die Unternehmung J g.44, Nr.2, S. 79-99.

Krulis-Randa, J .S. (1990) Globalisierung. In: Die Unternehmung J g.44, Nr.2, S. 74-78.

Kumar, B.N. (1988) Interkulturelle Managementforschung. In: WiSt, Heft 8, S. 389-394. 
Kumar, B.N./Karlshaus, M. (1992) Auslandseinsatz und Personalentwicklung. In: Zeitschrift für Personalforschung 6.J g. Heft 1, S. 59-75.

Landis, D./Brislin, R.W. (1983) Handbook of Intercultural Training, Vol. I - III, New York.

Laurent, A. (1986) The Cross-Cultural Puzzle of International Human Resource Management. In: Human Resource Management, Spring, Vol.25, No.1, S. 91-101.

Levitt, B./March, J.B. (1988) Organizational Learning. In: Annual Review of Sociology, Vol.14 S. 319-340.

Levitt, T. (1983) The Globalization of Markets. In: Harvard Business Review, May-J une, S. 92-102.

Lichtenberger, B. (1992) Interkulturelle Mitarbeiterführung. Überlegungen und Konsequenzen für das internationale Personal management. Stuttgart.

Lobel, S. (1991) Global Leadership Competencies: Managing to a Different Drumbeat, In: Human Resource Management, Vol. 29, No. 2, S. 39-47.

March, J./OIson, J. (1976) Organizational Learning and the Ambiguity of the Past. In: March, J ./Olson, J . Ambiguity and Choice in Organizations. Bergen, S. 54-67.

Meffert, H. (1989) Globalisierungsstrategien und ihre Umsetzung im internationalen Wettbewerb. In: Die Betriebswirtschaft 49 Nr.4, S. 445-463.

Mendenhall, M./Oddou, G. (1985) The Dimension of Expatriate Acculturation: A Review. In: Academy of Management Review, Vol.10, Nr.1, S. 39-47.

MendenhalI, M./Dunbar, E./Oddou, G. (1987) Expatriate Selection, Training and CareerPathing: A Review and Critique. In: Human Resource Management, Fall, Vol.26, Nr.3, S. 331-345.

Morical, K./Tsai, B. (1992) Adapting Training for Other Cultures. In: Training \& Development, April, S. 65-69.

Negandhi, A./Baliga, B. (1976) Quest for Survival and Growth. A Study of American, European, and J apanese Multinational Corporations. Berlin.

Negandhi, A. (1983) Cross-Cultural Management Research: Trend and Future Directions, In: J ournal of International Business Studies, Fall, S. 17-28.

Negandhi, A./Prasad, B. (1970) Comparative Management. New York.

Nomuri, M. (1992) Toyotismus am Ende? Dialog der IG Metall und der Hans-BöcklerStiftung. Frankfurt.

Ohmae, K. (1989) Managing the Borderless World. In: Harvard Business Review, MayJ une, S. 152-161.

Pautzke, G. (1989) Die Evolution der organisatorischen Wissensbasis. Bausteine zu einer Theorie des organisatorischen Lernens. München.

PerImutter, H.V. (1969) The Tourtuos Evolution of the Multinational Corporation. In: Columbia J ournal of World Business. Vol.4 No. 1, S. 9-18. 
Pieper, R. (Hrsg.) (1990) Human Resource Management. An International Comparison. Berlin, New York.

Porter M.E. (Hrsg.) (1989) Globaler Wettbewerb. Strategien der neuen International isierung. Wiesbaden.

Prahalad C.K. (1991) Globalization: The Intellectual and Managerial Challenges. In: Human Resource Management, Spring, S. 27-37.

Prahalad C. K. /Doz Y. (1987) The Multinational Mission: Balancing Local Demands and Global Vision. New-York.

Pucik, V. (1988) Strategic Alliances, Organizational Learning, and Competitive Advantage: The HRM Agenda. In: Human Resource Management, Spring, Nr.1, S. 77-93.

Ronen, S. (1986) Comparative and Multinational Management. New York.

Sackmann, S.A. (1990) Möglichkeiten der Gestaltung von Unternehmens-kultur. In: Lattmann (Hrsg.) Die Unternehmenskultur. Heidelberg, S. 153-187.

Sathe, V. (1983) Implications of Corporate Culture: A Manager's Guide to Action. In: Organizational Dynamics, Autumn, S. 13-28.

Sattelberger, T. (1989) Innovative Personal entwicklung. Wiesbaden.

Sattelberger, T. (1991) Die lernende Organisation. Wiesbaden.

Sattelberger, T. (1992) Die lernende Organisation. I m magischen Dreieck von Strategie-, Kultur- und Strukturentwicklung. In: Personalführung Nr.4, S. 286-295.

Schein, E.H. (1985) Organizational Culture and Leadership. San Francisco.

Schein, E.H. (1989) Organizational Culture: What it is and How to Change it. In: Evans, P./Doz, Y./Laurent, A. (Hrsg.) Human Resource Management in International Firms Change, Globalization, Innovation. Houndmills, u.a. S. 56-83.

Scheuch, R.W. (1985) Strategische Anpassung der Unternehmung. Zürich.

Schmidt, K. (1991) Corporate Identity in einem multikulturellen Markt. Wuppertal, London.

Schneider, S.C. (1988) National vs. Corporate Culture: I mplications for Human Resource Management. In: Human Resource Management, Summer Nr.2, S. 231-246.

Schön, D. (1983) The Reflective Practitioner. New York.

Schulte, C. (1988) Personalstrategien für multinationale Unternehmen. In: Zeitschrift für Personalforschung, J g.2 Heft 3, S. 179-195.

Shrivastava, P. (1983) A Typology of Organizational Learning Systems. In: J ournal of Management Studies, Nr.20, S. 7-28.

Siebold, H. (1992) Japanisches Management in der Bundesrepublik Deutschland - Eine Analyse zum Personalmanagement auf $\mathrm{Führungsebene} \mathrm{in} \mathrm{japanischen} \mathrm{Niederlassungen}$ in der Bundesrepublik. Diplom arbeit Konstanz. 
Staehle, W.H. (1990) Human Resource Management and Corporate Strategy. Pieper, R. (Hrsg.) Human Resource Management. An International Comparison. Berlin, New York. S. 27-41.

Staehle, W.H. (1991) Redundanz, Slack und lose Kopplung in Organisationen: Eine Verschwendung der Ressourcen? In: Staehle, W.H./Sydow, J. (Hrsg.) Managementforschung 1, Berlin, New York. S. 313-347.

Steinmann H./Schreyögg G. (1991) Management, 2. Aufl. Wiesbaden

Thomas, A (1990) Interkulturelles Handlungstraining als Personalentwicklungsmaßnahme. In: Zeitschrift für Arbeits- und Organisationspsychologie, 34/3, S. $149-154$.

Vahlefeld ， H.W. (1992) J apanische Herausforderung ohne Ende. Stuttgart.

Vivelo, F.R. (1981) Handbuch der Kulturantropol ogie. Stuttgart.

Weick, K.E. (1986) Organizations as Cognitive Maps: Charting Ways to Success and Failure. In: Bennis, W./Mason, R./Mitroff, I. (Hrsg.) The Thinking Organization. San Francisco, London.

Wilkins, A.L./Ouchi, W.G. (1983) Efficient Cultures: Exploring the Relationship between Culture and Organizational Performance. In: Administrative Science Quarterly 28, S. 468-481.

Womack, J.P./J ones, D.T./Ross, D. (1992) Die zweite Revolution in der Automobilindustrie. Frankfurt, New York. 